\title{
Utilization of an ultrasound beam steering angle for measurements of tissue displacement vector and lateral displacement
}

This article was published in the following Dove Press journal:

Reports in Medical Imaging

13 September 2010

Number of times this article has been viewed

\section{Chikayoshi Sumi}

Department of Information and Communication Sciences, Faculty of Science and Technology, Sophia University, Tokyo, Japan
Correspondence: Chikayoshi Sumi Department of Information and Communication Sciences, Faculty of Science and Technology, Sophia University, 7-I, Kioi Cho, Chiyoda Ku, Tokyo 102-8554, Japan

Tel $+8|-3-3238-34| 5$

Fax +8I-3-3238-332I

Email c-sumi@sophia.ac.jp
Abstract: A number of ultrasonic displacement/velocity measurement methods have been extensively developed for measurements of blood flow, tissue motion, and strain. Lateral modulation (LM) methods have also been reported using steered, crossed beams, and these methods permit measurements of displacement vectors. In this report, a new beam steering method for the transmission and reception of ultrasound is proposed, which can enable measurements of lateral displacements and of arbitrary displacement vectors with a very high degree of accuracy. Because this beam steering method uses only a steering angle, this method is referred to as ASTA. With ASTA, the number of available methods to obtain a displacement vector measurement is limited to previously developed block-matching methods, such as the multidimensional cross-spectrum phase gradient method, and the multidimensional autocorrelation method (MAM) and the multidimensional Doppler method (MDM) using a block-matching method (the methods using block matching are referred to as $\mathrm{MAMb}$ and $\mathrm{MDMb}$, respectively). Being dependent on the measurement method, only a lateral displacement measurement can be made even if the methods are multidimensional, ie, previously developed MAM and MDM using a moving average and a mirror setting of the obtained steered beams, and one-dimensional (1D), such as an autocorrelation method. Considerations of beamforming schemes using LM and ASTA show that the simple ASTA beamforming method increases capabilities for real-time measurements and requires a small physical aperture when compared with LM. For lateral displacement measurements (eg, blood flow in a carotid artery), a lateral coordinate must correspond to the direction of the target's lateral motion, and the steering angle used is as large as possible to increase the measurement accuracy of a lateral displacement. However, for displacement vector measurements to describe complex tissue motions (eg, cardiac motion), if the axial coordinate corresponds to the depth direction in the target tissue, an ideal steering angle will be $45^{\circ}$ A two-dimensional echo simulation shows that for the block-matching methods, LM yields more accurate displacement vector measurements than ASTA, whereas with MAM and MDM using a moving average and a mirror setting and 1D methods, ASTA yields more accurate lateral displacement measurements than LM. The block-matching method requires fewer calculations than the moving average method; however, a lower degree of accuracy is obtained. As with LM, multidimensional measurement methods yield more accurate measurements with ASTA than the corresponding 1D measurement methods. Summarizing, for displacement vector measurements or lateral displacement measurements using the multidimensional measurement methods, the ranking of the degree of measurement accuracy and stability is ASTA with a mirror setting $>$ LM with a moving average $>$ LM with block matching $>$ ASTA with block matching. Because every tissue has its own motion (heart, liver, etc) and occasionally obstacles, such as bones, interfere with the measurements, the target tissue will determine the selection of the proper beamforming method with a choice between LM and ASTA. As for use with LM previously clarified, an appropriate displacement measurement method should also be selected for use with ASTA according to the echo signal-to-noise ratio, a required spatial resolution and a required calculation speed. ASTA, together with LM, can potentially enable the utilization of new aspects of displacement measurements.

Keywords: a steering angle, lateral modulation, displacement vector measurement, lateral displacement measurement submit your manuscript $\mid$ www.dovepress.com

Dovepress

DOI: 10.2147/RMI.SII 407
Reports in Medical Imaging 2010:3 6I-8I

(C) 2010 Sumi, publisher and licensee Dove Medical Press Ltd.This is an Open Access article which permits unrestricted noncommercial use, provided the original work is properly cited. 


\section{Introduction}

Various ultrasonic (US) displacement/velocity measurement methods have been extensively developed for the measurements of blood flow (eg, the continuous wave Doppler method $[\mathrm{DM}],{ }^{1}$ the pulse wave $\mathrm{DM},{ }^{2}$ the autocorrelation method $[\mathrm{AM}],{ }^{3}$ the cross-correlation method [CCM]), ${ }^{4}$ tissue strain (eg, DM, $\mathrm{AM},{ }^{5} \mathrm{CCM}^{6}$ ), and sonar data and other target motions. These measurement methods produce an axial displacement/velocity/strain measurement (ie, they are one-dimensional [1D] measurement methods), whereas other development results in measurements of multidimensional displacement/velocity vectors for blood flow $^{7}$ and for tissue motion vectors ${ }^{8}$ (ie, multidimensional cross-correlation method [MCCM]).

For both applications, tissue motion and blood flow measurements, we developed multidimensional displacement vector measurement methods, which can permit simultaneous axial and lateral displacement measurements, ie, the multidimensional cross-spectrum phase gradient method (MCSPGM), ${ }^{9,10}$ the multidimensional autocorrelation method (MAM), and the multidimensional Doppler method (MDM). ${ }^{11-13}$ A displacement vector measurement method similar to MAM has also been reported by another group. ${ }^{14}$

Specifically, with MCSPGM, local echo phase characteristics (ie, the phase of the local cross-spectrum evaluated from local region echo data) are used under the assumption that the local region has a rigid motion (ie, block matching is performed), whereas with MAM and MDM, an instantaneous phase moving average is used. Also with MAM and MDM, block matching can be performed by applying a least squares estimation to simultaneous equations obtained in a local region or in a window using the same assumption ${ }^{11-13}$ (these methods will be referred to as MAMb and MDMb, respectively). As shown with simulations, ${ }^{13}$ both MAM and MDM yield accurate measurements comparable to those obtained with MCSPGM; however, they require less computational time (in particular, MDM) and can provide real-time measurements. The degree of accuracy of these measurement methods depends on the echo signal-to-noise ratio (SNR) and the required spatial resolution, and they have been summarized together with their calculation speeds in a study by Sumi. ${ }^{13}$ The spatial resolution is determined by the size of the local region or local window used for block matching, or by the moving average of an instantaneous phase difference and an instantaneous frequency. Although MCCM also yields a displacement vector using block matching, the method requires numerical interpolation (using cosines, parabolic functions, etc) of the cross-correlation function to yield analog displacement vector data. However, the MCSPGM, ${ }^{9,10}$ MAM, and MDM ${ }^{11-13}$ do not require such interpolation. Thus, these methods do not suffer from any artifacts due to approximation errors produced by such interpolation calculations.

In general, however, the accuracy of lateral displacement measurements is significantly lower than the accuracy of axial displacements, ${ }^{9,10,15}$ and even if the target moves or deforms primarily in the lateral direction, the simultaneous measurements result in an accurate measurement of axial displacement. ${ }^{16}$ Thus, by developing lateral modulation (LM) methods, ${ }^{11-13,17-21}$ the accuracy of measurements of lateral displacements improved, along with measurements of axial displacements. Thus, these methods make it possible to deal with a variety of tissues, and in particular, deeply situated tissues, and tissues with motion, such as the heart and liver, which are difficult to deal with using noninvasive methods.

In the medical US field, initial LM applications ${ }^{22}$ were applied to generate measurements of blood flow in blood vessels (eg, in the carotid artery) running parallel to the surface of the body (Jensen ${ }^{23}$ and Anderson ${ }^{24}$ ). Subsequently, measurements of tissue strain were achieved by my group ${ }^{17}$ using multidimensional displacement vector measurement methods, such as MCSPGM. Alternatively, for blood flow ${ }^{23-26}$ and other tissue strain $^{27}$ measurements, measurements of axial and lateral displacements were achieved using 1D measurement methods, such as 1D AM by using point spread functions (PSFs) oscillating only in the lateral and axial directions (ie, ones obtained by demodulations ${ }^{25,26}$; a newly developed demodulation method is also described in Appendix A in this report). However, such measurements using 1D methods suffer from decorrelation of the echo signals due to displacements orthogonal to the oscillation direction. Also, because the strain tensor is obtained by differentiating the measured displacement vector components using a differential filter (ie, a type of high-pass filter) ${ }^{28}$ the displacement vector must be measured with a considerably high accuracy.

A high accuracy in measuring target motions can be realized with the combined use of $\mathrm{LMs}^{11-13,17-21}$ and displacement vector measurement methods, ${ }^{9-13}$ which allow simultaneous axial and lateral displacement measurements (ie, MCSPGM, MAM, and MDM). Jensen ${ }^{23}$ described an apodization function that was obtained by a Fraunhofer approximation of a lateral rectangular envelope PSF (ie, a designed lateral PSF), which is expressed by ringing sinc functions. Recent reports from my group ${ }^{12,13,17}$ described 
a new useful modulation technique (the lateral Gaussian envelope cosine modulation method) that used band-limited, modulated spectra by using a lateral Gaussian envelope PSF, in which suitable focusing was also performed (ie, spherical focusing). Thus, for strain tensor measurements and tissue shear modulus reconstructions, obtaining comparable highly accurate measurements for axial and lateral displacements require that the LM frequency and lateral bandwidth be significantly increased ${ }^{17,18}$ compared with the values observed in other reported measurements of blood flow ${ }^{25,26}$ and tissue strain. ${ }^{27}$ The modulation frequency achieved was $3.75 \mathrm{MHz}$ when the US frequency was $7.5 \mathrm{MHz}$.

In considering energy consumption, a more suitable apodization function was found, which uses power functions instead of Gaussian functions. ${ }^{18,19,29} \mathrm{On}$ an agar phantom, the apodization function also enabled LM echo imaging with almost the same lateral resolution as axial resolution. Using simulations, a proper PSF was found, which had a wide full width at half maximum and short feet, such as those seen with the envelope of a power function. ${ }^{20}$ Optimization methods were further developed for realizing such a PSF using linear ${ }^{21}$ and nonlinear ${ }^{20}$ methods. The proper PSFs will be reported in detail elsewhere. ${ }^{30}$

These LMs can also be resolved simply by using a superposition of steered, crossed beams. ${ }^{13,18,19}$ That is, these LMs can also be performed by the superposition of multiple steered beams with different steering angles obtained with the multiple transmission method (MTM) or synthesized from a set of received echo data using the multidirectional synthetic aperture (SA) method (MDSAM). With this type of beamforming, multiple transducers can also be used (ie, using simultaneous or successive transmissions/receptions of US). The most accurate results of a shear modulus reconstruction and a strain tensor measurement were obtained on an agar phantom ${ }^{18}$ with the parabolic modulation using a spherical focusing (Method 1) with $2 \lambda$ (see Table 8 in Sumi et $\mathrm{al}^{18}$ ). A different result is obtained only for the mean value of the relative shear modulus of the stiff inclusion with respect to the surrounding region: 3.28 (MDSAM) vs 3.29 (MTM).

Originally, with $\mathrm{MTM}^{31-34}$ and MDSAM, ${ }^{33-35}$ only the most accurately measured axial displacements from the respective beams could be used to obtain a displacement vector (ie, there is no superposition of beams). Although 1D measurement methods can also be used ${ }^{31-35}$ in place of the multidimensional measurement methods, ${ }^{33,34}$ the same decorrelation of local echo signals (mentioned above) occurs due to target displacement in a direction orthogonal to the beams. ${ }^{9,13}$ Thus, the 1D measurement methods will result in a lower measurement accuracy for axial displacement than the corresponding multidimensional measurement methods. ${ }^{33,34}$ To mitigate echo decorrelation which can also affect multidimensional methods, our previously developed phase matching method based on a spatial shift and compression/stretching is used (ie, displacements and strains measured by MCCM or MCSPGM are used to search for corresponding local echo data), ${ }^{9,10,13}$ and the phase matching also permits accurate freehand in vivo measurements of axial and lateral displacements. ${ }^{36}$ That is, the phase matching succeeded in coping with target motions outside of the beams, which had classically made it impossible to measure even the axial displacement for the imaging of axial strain and blood flow. Similar phase matching was also achieved by another group, ${ }^{37}$ which used local 1D-rf echo data to search for the corresponding local echo data in a lateral direction with a 1D CCM. However, this approach required extensive calculations for the correlations and used laterally interpolated 1D rf-echo data.

Because MDSAM requires less US data acquisition time than MTM, if transmitted ultrasound energies are sufficient, beamforming will suffer less from tissue motion artifacts. To obtain high intensity transmitted ultrasound signals, a virtual source can be used (Appendix B). ${ }^{38,39}$ However, if tissue motion artifacts do not occur, MTM yields more accurate measurements. ${ }^{13,18,19,33,34}$ Under conditions in which motion artifacts do not occur, comparisons of the measurement accuracies of axial and lateral displacements achieved with superposition or no superposition of beams was analyzed geometrically with respect to the various angles between the beams. ${ }^{13}$ However, for practical beamforming applications, the echo SNRs from steered beams must also be considered (ie, an overly large steered angle makes the echo SNR low). ${ }^{18,19}$

In this report, a simpler beamforming method is proposed for measuring a displacement vector or a lateral displacement. ${ }^{39,40}$ Beamforming is performed based on the steering of beams with a defined steering angle. By using the new beamforming method instead of the above-mentioned beamforming methods, more accurate and more rapid measurements can be realized except when using blockmatching methods, such as MCSPGM, MAMb, MDMb, and MCCM. The new beamforming method is referred to (and abbreviated) as ASTA (ie, a steering angle) (Figure 1).

In this report, first, the new beamforming method (ASTA) is described, and in simulations, the measurement accuracies of the axial and lateral displacements obtained with ASTA are compared with those obtained with the corresponding LM 


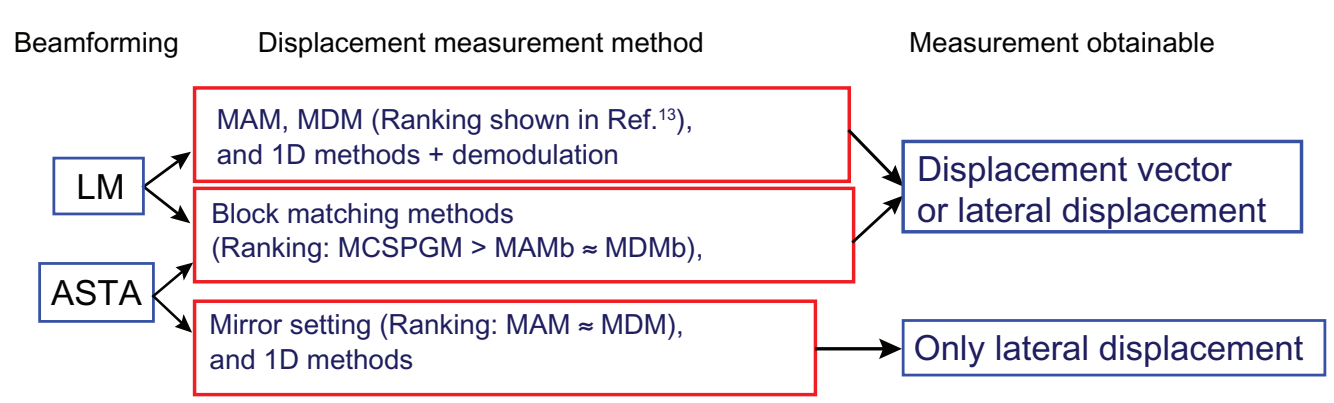

\author{
Ranking: For multidimensional methods, \\ ASTA with mirror setting $>$ LM with moving average \\ $>$ LM with block matching > ASTA with block matching. \\ For 1D methods, \\ ASTA > LM. \\ Corresponding multidimensional method > ID method.
}

Figure I Flow chart about the beamforming of LM or ASTA (a steering angle), displacement measurement method usable (ie, MCSPGM [ie, using block matching], MAM and MDM using moving average, the corresponding ID methods, MAMb and MDMb, and MAM and MDM using mirror setting with moving average but no block matching), and displacement obtainable (ie, displacement vector or lateral displacement). Ranking of measurement accuracies and stabilities is also shown.

Abbreviations: LM, lateral modulation; MCSPGM, multidimensional cross-spectrum phase gradient method; MAM, multidimensional autocorrelation method; MDM, multidimensional Doppler method; ID, one dimensional; MAMb and MDMb, MAM and MDM using block matching.

method. With the LM method, laterally symmetric beams are superimposed on the steered beams obtained by ASTA. Finally, discussions and conclusions are provided.

\section{Beam steering with a steering angle (ASTA) Beamforming for ASTA}

With ASTA (Figure 1) for a 2D region of interest (ROI) expressed using the Cartesian coordinate system $(x, y)$ as shown in Figure 2a (left), steered beams with a steering angle $\theta$ are formed (ASTA, $0<\theta<\pi / 2[\mathrm{rad}]$ ). The direction of the steered beam is expressed using the coordinate $s$ that is orthogonal to the coordinate $t$. For proper beamforming, apodization and focusing are performed for transmission and dynamic reception focusing (ie, electrically).

By slanting a linear 1D-array transducer, the ASTA can also be generated mechanically. If a large steering angle cannot be obtained electrically, the mechanical steering is also used together. When mechanical steering is performed, the orthogonal, axial, and lateral directions of a coordinate system should be set such that the axial direction should coincide with the depth in a target tissue by rotating the coordinate system when beamforming, after receiving echo signals for a $\mathrm{SA}$, or after obtaining steered beams (Appendix C). A sensor to detect the mechanical steering angle may be required.

For a 3D ROI, similarly, such steered beams with a steering angle can also be realized by using a 2D-array transducer (Figure 2a, right). Specifically, the steered beams are slanted in both the lateral and elevational directions.
As shown in Figure 2b, a single quadrant (left) and single octant (right) spectra are obtained for the 2D and 3D ROIs, respectively. In both cases, the same spectra are obtained symmetrically. In the illustrated 2D spectra (2b, left), for instance, an increase in the steering angle $\theta$ increases the lateral frequency $f_{y}$ and decreases the axial frequency $f_{x}$ from the US frequency, and vice versa, because with respect to the US frequency $f_{0}$,

$$
f_{x}=f_{0} \cos \theta \text { and } f_{y}=f_{0} \sin \theta .
$$

Thus, in general, the measurement accuracies of the axial and lateral displacements, respectively, become lower and higher, and vice versa.

This occurs because theoretically, the coordinates of the spectra also rotate with $\theta$. That is, when the spatial coordinates $x, y, s$, and $t$ have the following relations (see the rotation of the coordinates by $\theta$ in Figure $2 \mathrm{a}$, left),

$$
\left(\begin{array}{l}
x \\
y
\end{array}\right)=\left(\begin{array}{cc}
\cos \theta & -\sin \theta \\
\sin \theta & \cos \theta
\end{array}\right)\left(\begin{array}{l}
s \\
t
\end{array}\right) .
$$

The corresponding Fourier's coordinates $X, Y,(S$, and $T$ ) have the same geometrical relations (Figure 2b, left) as follows:

$$
\left(\begin{array}{l}
X \\
Y
\end{array}\right)=\left(\begin{array}{cc}
\cos \theta & -\sin \theta \\
\sin \theta & \cos \theta
\end{array}\right)\left(\begin{array}{l}
S \\
T
\end{array}\right)
$$

The relations can be guaranteed because the inverse $2 \mathrm{D}$ Fourier's transform $f(x, y)$ of $2 \mathrm{D}$ spectra $F(X, Y)$ satisfies the following equations using Eqs. 2 and 3, 


$$
\begin{aligned}
f(x, y) & =\int_{-\infty}^{\infty} \int_{-\infty}^{\infty} F(X, Y) \exp [2 \pi(X x+Y y)] d X d Y \\
& =\int_{-\infty}^{\infty} \int_{-\infty}^{\infty} F(X, Y) \exp [2 \pi(X(s \cos \theta-t \sin \theta)+Y(s \sin \theta+t \cos \theta))] d X d Y \\
& =\int_{-\infty}^{\infty} \int_{-\infty}^{\infty} F(X, Y) \exp [2 \pi((X \cos \theta+Y \sin \theta) s+(-X \sin \theta+Y \cos \theta) t)] d X d Y \\
& =\int_{-\infty}^{\infty} \int_{-\infty}^{\infty} F(S \cos \theta-T \sin \theta, S \sin \theta+T \cos \theta) \exp [2 \pi(S s+T t)]|J(S, T)| d S d T \\
& =\int_{-\infty}^{\infty} \int_{-\infty}^{\infty} F(S \cos \theta-T \sin \theta, S \sin \theta+T \cos \theta) \exp [2 \pi(S s+T t)] d S d T \\
& =f(s \cos \theta-t \sin \theta, s \sin \theta+t \cos \theta),
\end{aligned}
$$

where $|\mathrm{J}(\mathrm{S}, \mathrm{T})|$ is the determinant of Jacobian matrix $J(S, T)$, ie,

$$
\begin{aligned}
|J(S, T)| & =\left|\begin{array}{ll}
\frac{\partial X}{\partial S} & \frac{\partial Y}{\partial S} \\
\frac{\partial X}{\partial T} & \frac{\partial Y}{\partial T}
\end{array}\right| \\
& =\left|\begin{array}{ll}
\cos \theta & \sin \theta \\
-\sin \theta & \cos \theta
\end{array}\right| \\
& =1
\end{aligned}
$$

This is also for 3D spectra (the corresponding equations are not shown).

For real-world US beamforming, however, the increase in $\theta$ does not make the axial and lateral frequencies so low and high compared with those determined geometrically by the rotation of the spectra, respectively (ie, Eq. [1]), and an overly large $\theta$ yields a low echo SNR. ${ }^{18,19}$

In contrast, with LM (Figure 1) for a 2D ROI, when laterally symmetric steered beams are crossed as shown in Figure 2c (left), the spectra shown in Figure 2c (right) are obtained, ie, 2 independent quadrant spectra. For a 3D ROI, 3 or 4 independent octant spectra are obtained. ${ }^{13}$ However, similarly to ASTA, the steering angles are set to be as large as possible.

Both beamformings can also be performed for arbitrary orthogonal coordinate systems such as orthogonal curvilinear coordinates and the following displacement measurements can be performed.

\section{Displacement vector measurements}

When using block-matching methods, such as MCSPGM, MAMb, MDMb, and MCCM, for both types of beamforming (ie, ASTA and LM), a 2D or 3D displacement vector measurement can be obtained. When using LM, a displacement vector can also be measured by using MAM and MDM with no block matching but with a moving average (ie, MAM and MDM) and a combination of 1D methods and demodulation methods, ${ }^{25,26}$ or a newly developed demodulation method (Appendix A) (Figure 1).

As shown in the following section, for a displacement vector measurement using $\mathrm{LM}, \mathrm{MAMb}$, and $\mathrm{MDMb}$, respectively, yield lower accuracy measurements than MAM and MDM, although MAMb and MDMb, respectively, permit more rapid calculations. For ASTA and LM, the measurement accuracies are also compared in the following section. As will be shown, the ranking of measurement accuracy is

LM with a moving average $>$ LM with block matching $>$ ASTA with block matching.

It has been shown that $1 \mathrm{D}$ measurement methods with LM yields less accurate measurements than the corresponding multidimensional methods. ${ }^{13}$ For the demodulation required, a newly developed method yields the most accurate results of all demodulation methods (Appendix A). For displacement vector measurements, only the attachment of an US transducer onto the target surface is required.

\section{Lateral displacement measurements}

When using ASTA for a lateral displacement measurement, the above-mentioned displacement vector measurement methods together with ASTA can be used at least, ie, blockmatching methods, such as MCSPGM, MAMb, MDMb, and MCCM. In addition, by performing the next described quasiLMs, multiple equations can be obtained, and then MAM and MDM without block matching can also be used, although axial displacements cannot be measured (Figure 1).

As shown in Figure 3a, by setting the axially or laterally symmetric spectra (referred to as a mirror setting) specifically at the position A or B, a rf image superposed by the original rf image and the axially (Figure 3b, left) or laterally (right) 

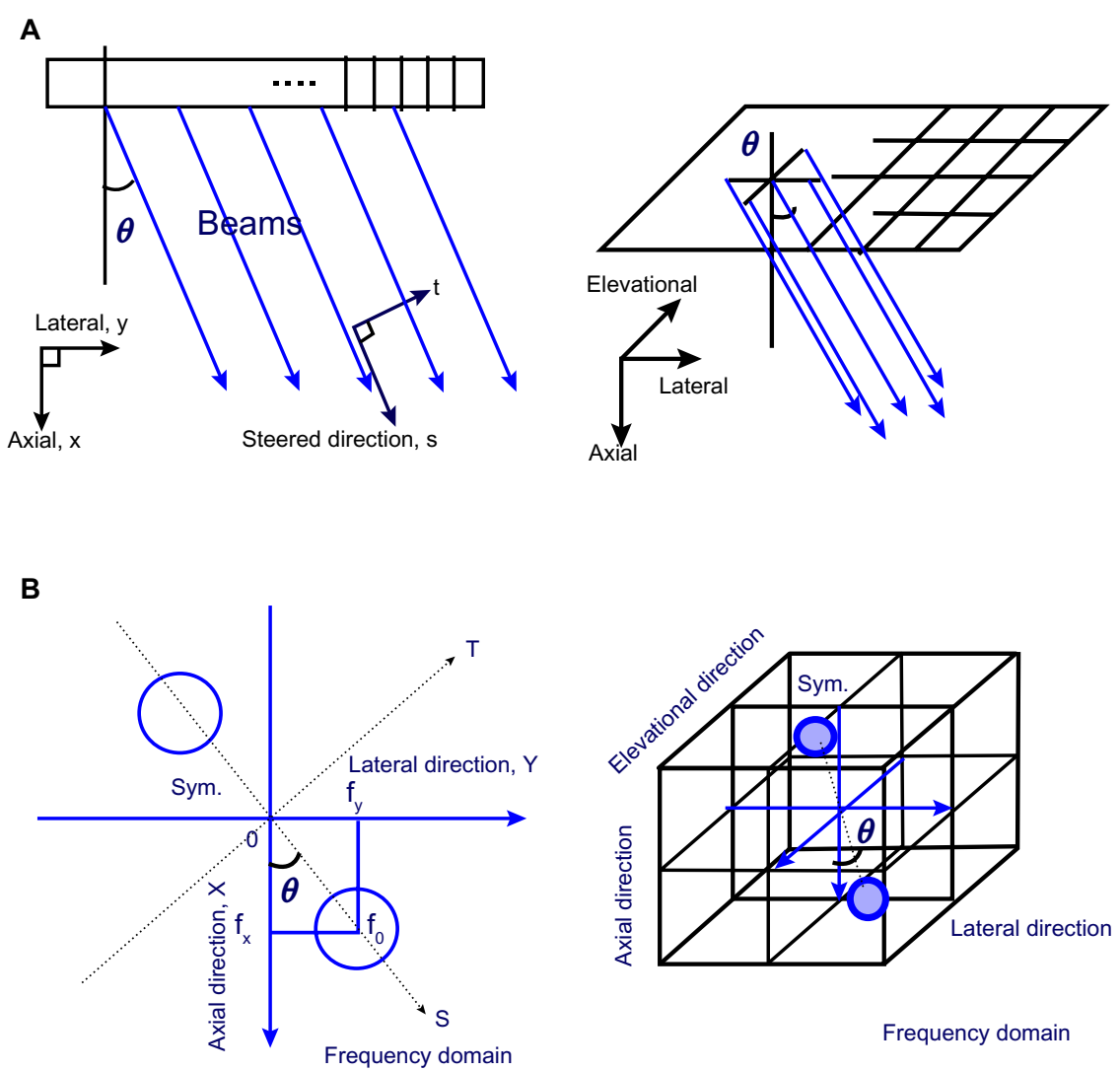

C
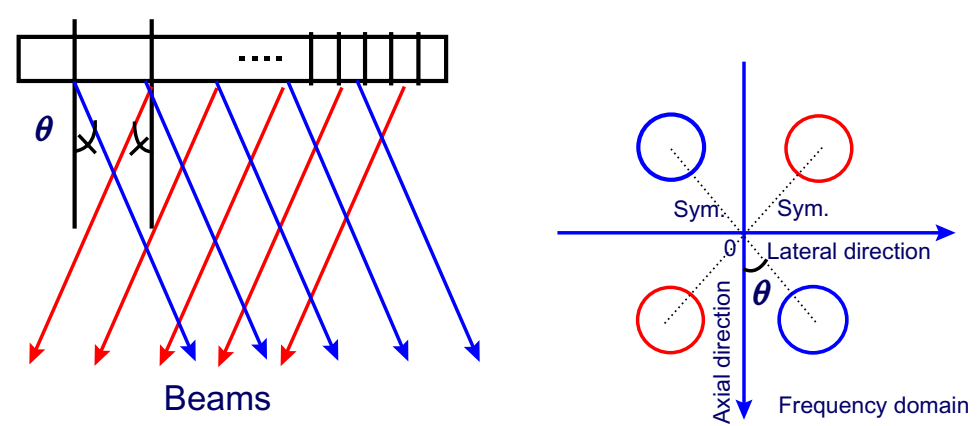

Figure 2 Beamforming using steering. A) Steered beams with a steering angle (ASTA) obtained using ID-array (left) and 2D-array (right) transducers. B) Spectra obtained by ASTA using the ID-array (left) and 2D-array (right) transducers shown in (a). C) Crossed beams (left) and spectra (right) obtained by LM using a ID-array transducer. Those obtained using a 2D-array transducer is omitted (see Sumi' ${ }^{3}$ ).

Abbreviations: ID, one dimensional; 2D, two dimensional; LM, lateral modulation.

inverted rf image are obtained. For a steered beam or local steered beams, by using the mirror setting in a frequency domain or superposing the inverted beam in a spatial domain, a quasi-LM can be performed. However, the target measurement is limited to only the axial or lateral motion. When the target moves in an axial direction, conventional axial displacement measurement methods (ie, 1D measurement methods, such as $1 \mathrm{D} \mathrm{AM}^{1-6}$ ) can be used, and then a quasiLM is performed as shown in Figure $3 \mathrm{~b}$ (left) for a lateral displacement measurement using MAM or MDM. For a lateral displacement measurement, ASTA with the mirror setting yields the most accurate measurements (see simulations in following section). Although all block-matching methods can also be used for such a quasi-LMs, these cases are not dealt with in this report, because the measurement accuracy is low. As will be shown, the ranking of measurement accuracies is

$$
\begin{aligned}
& \text { ASTA with a mirror setting }>\text { LM with a moving } \\
& \text { average }>\text { LM with block matching }>\text { ASTA with a } \\
& \text { block matching. }
\end{aligned}
$$

Such a lateral displacement measurement is a very important application for ASTA, eg, in measuring blood flow in a vessel running in a direction almost parallel to the body surface as shown in Figure 3c, such as in a carotid artery. For such measurements, a lateral coordinate must correspond to 
A

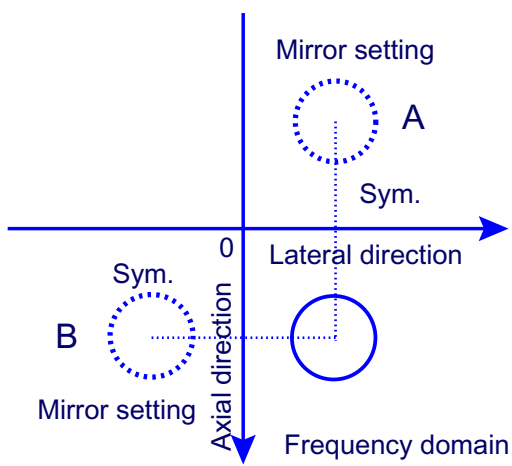

B
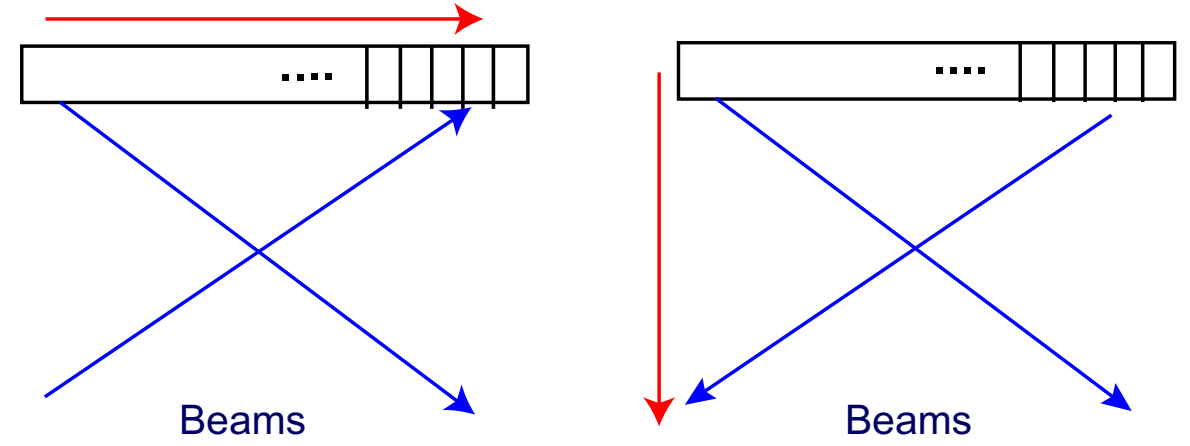

C
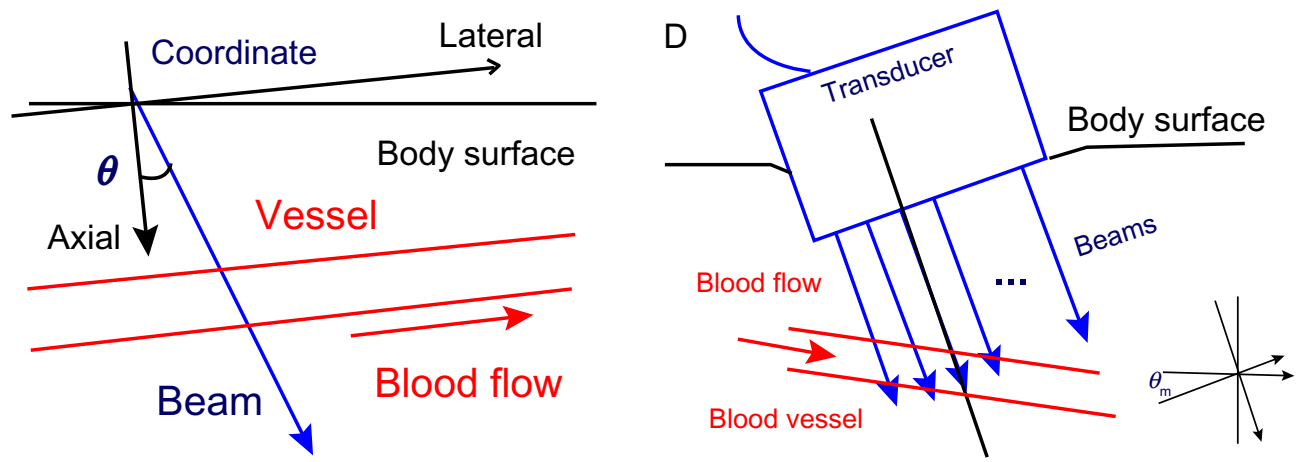

Figure 3 Lateral displacement measurements. A) Mirror-setting of spectra for a 2D ROI, ie, at position A or B. B) Quasi-crossed beams obtained by superposing an original beam and an axially (left) or laterally (right) inverted beam. These are also realized by a mirror-setting in a frequency domain as shown in (a), ie, at positions $A$ (left) and B (right), respectively. C) The coordinate system is set so that the direction of a lateral coordinate should correspond to that of a target motion, ie, blood flow in a vessel that runs almost in a lateral direction. D) A mechanically steered beam for which the direction (axial direction) should correspond to the target motion, although in most cases, the directions cannot coincide. Such a steered beam can also be realized electrically. Abbreviation: 2D ROI, two-dimensional region of interest.

the direction of the target lateral motion to increase measurement accuracy, although usually, the direction of the beam should coincide with that of the motion by slanting an US transducer manually, or electrically (also a new method), particularly, when using 1D methods ${ }^{1-6}$ (Figure 3d). In most cases, it is impossible to position the direction of beams to completely coincide with that of the measured motion, and
ASTA and LM will produce a more accurate measurement of lateral motion than the conventional 1D measurements. Thus, although LM permits the measurement of an arbitrary motion absolutely, being dependent on the measurement method, ASTA permits an accurate measurement of a lateral motion only, ie, by MAM and MDM using mirror settings. However, the use of ASTA permits a simpler manual technique 
of obtaining measurements for medical doctors or clinical staffs than the conventional 1D measurement technique. Ideally, a sensor for a mechanical steering angle and an automatic detector for blood vessels will also be useful. As with displacement vector measurements, the coordinate system must also be properly chosen before or after beamforming (Appendix C).

With ASTA, when an axial displacement occurs simultaneously with a lateral displacement or an elevational displacement, measurement errors can occur. For example, for an unknown 2D displacement vector $(d x, d y)$, this occurs because the equation with $(d x, d y)$ can be expressed at each position in a 2D ROI using axial and lateral instantaneous frequencies $(f x, f y)$, and the instantaneous phase differences $c$ between premotion and postmotion are obtained from the original quadrant spectra as follows:

$$
f x d x+f y d y=c
$$

and the mirror setting of the spectra yields the following equation,

$$
-f x d x^{\prime}+f y d y=c .
$$

Because $d x^{\prime}=-d x$, the obtained Eq. (8) is equal to equation (8)'. However, if $d x=0$, solving the simultaneous Eqs. (8) and (8)' under the condition that $d x=d x^{\prime}=0, d y$ is obtained as $c / f y$. However, if $d x \neq 0$, and Eq. (8)' is solved using $\left(d x^{\prime}, d y\right)$ for $(d x, d y)$, a measurement error can occur.

The use of 1D measurement methods, such as 1D AM, 1D DM, 1D CSPGM, and 1D CCM with ASTA as well as with LM, ${ }^{13}$ should also be mentioned because such methods permit rapid calculations (Figure 1). For 1D methods, without performing the quasi-LM or the demodulation, a lateral displacement can be measured as $c / f y$. However, the phase matching and moving average or least squares estimation should be performed using a local 2D or 3D window or region. ${ }^{13}$ In the following section, the measurement accuracy of a lateral displacement is compared with measurements obtained with MAM and MDM together with those obtained with LM instead of ASTA. Interestingly, ASTA yields a higher measurement accuracy than LM. That is, for 1D methods, the ranking of measurement accuracies and stabilities is

$$
\text { ASTA }>\text { LM with a demodulation. }
$$

Moreover, as with LM, 1D methods also yield less accurate measurements with ASTA than the corresponding multidimensional methods.
If an axial displacement $d x$ is not zero, the measurement error is $-(f x / f y) \times d x$. As in the case for MAM and MDM, to increase the measurement accuracy of a lateral displacement measurement, a lateral coordinate should correspond to the direction of the target motion. Nevertheless, by steering beams in the direction of the target motion and using a spectra with a negative axial frequency instead of that with a positive axial frequency, the summation of weighted displacement components can be obtained as $(f x / f y) \times d x+d y$. If steering with a steering angle of $45^{\circ}$ can be performed, under the condition that $f x=f y$, one can accurately measure the summation of the displacement components, ie, $d x+d y$.

The above-described beamforming method for 2D measurements can easily be extended for 3D methods.

\section{Comparison of ASTA and LM}

As described above, LM enables a displacement vector measurement by using MCSPGM, MAM, MDM, and a combination of demodulation methods and the above-mentioned 1D measurement methods. However, ASTA enables only a lateral displacement measurement by using MAM, MDM, and 1D measurement methods, although a displacement vector measurement can be performed by using blockmatching methods, such as MCSPGM, MAMb, MDMb, and MCCM. For lateral displacement measurements using ASTA, because a uniform PSF can be realized at every depth, the measurement error that occurs in a displacement vector measurement due to a spatially variant PSF does not occur. With echo imaging, ASTA yields only a quasi-LM image. Overall, the number of applications of ASTA may be fewer than the number available for LM.

Before performing comparisons of measurement accuracy using the displacement vector measurement methods and 1D measurement methods with beamforming with ASTA and LM in simulations (following section), the quality of the beams formed by ASTA and LM are compared.

With LM, the following properties may lead to the deterioration of measurement accuracy:

1. For a measurement in a $2 \mathrm{D}$ or $3 \mathrm{D}$ ROI, when a classical SA is used, ${ }^{13,18,19}$ the US intensity transmitted from an element is small, which may yield low SNR echo data.

2. Alternatively, when crossed beams are superimposed, although a large US intensity can be obtained, time differences between the transmission of the beams can cause measurement errors, if the displacement occurs during these time differences.

3. If multiple beams that have different paths are used, the inhomogeneity of tissue properties affects beamforming. 
Specifically, propagation speed affects focusing (ie, the beam-crossing position), whereas attenuation and scattering lead to different frequencies of the crossed beams.

4. At the minimum, more time is required to complete a beamforming than that required with ASTA. Occasionally, more time is also required to complete a displacement calculation than is required with ASTA.

5. If obstacles, such as bone, exist in a superficial region, a deeply situated tissue cannot be dealt with because a larger physical aperture is required than for conventional beamforming.

In contrast, with ASTA, although used for a displacement vector measurement, the number of available methods is limited. Being dependent on the measurement method, only a lateral displacement measurement can be performed, and any of the above concerns, points $1-5$, will not become a problem, and a simple beamforming increases the ability to make real-time measurements together with higher accuracy in displacement measurements.

However, when using LM, point (1) can be managed with a virtual source (Appendix $\mathrm{B}^{38,39}$ ), ie, a larger intensity ultrasound signal can be obtained than with a classical SA. Moreover, point (5) will also be mitigated by using a recently developed virtual receiver (Appendix B). Otherwise, if a transmitted US intensity is large and the effects on US from tissue inhomogeneities and tissue motion are small, then LM yields a higher echo SNR than ASTA owing to more summations of echo signals. Thus in practical applications, a proper beamforming method should be selected for every organ and tissue because every tissue has its own motion (heart, liver, etc). Being dependent on the echo SNR, the required spatial resolution (determined by the local window/local region used), and the required calculation speed, as with $\mathrm{LM},{ }^{13}$ a proper measurement method can also be selected for ASTA. This will also be confirmed briefly in the following section.

If a displacement vector is measured using ASTA together with MAM and MDM or 1D methods, additional steering beams with different steering angles must be formed as with LM, ie, for 3D and 2D displacement vector measurements, 2 and 1 additional steering beams are required, respectively. For ASTA and LM, to increase the measurement accuracy of a lateral displacement, a high lateral frequency must be achieved by increasing the steering angle ${ }^{13}$ (Appendix C). When the pitch of the element or beam is not small enough, an aliasing of spectra occurs. This problem can be resolved by making the beam pitch appropriately small. Otherwise, the beams are interpolated in a frequency domain by increasing the lateral bandwidth padded by zeros.

\section{Simulations for ASTA and LM}

Simple simulations to test lateral displacement measurements were performed. Echo data were simulated by convolving white noise data in a 2D ROI with a Gaussian-type PSF with a spatially isotropic standard deviation (SD) $\sigma$ of $0.4 \mathrm{~mm}$ (see Sumi $\left.{ }^{10,13}\right)$. For the lateral motion $(0.01 \mathrm{~mm})$, the steering angle $\theta$ for ASTA was set at $45^{\circ}$ (Figure $2 a$ ). Thus, the PSF is expressed by

$\operatorname{PSF}(x, y)=\exp \left(-\frac{1}{2 \sigma^{2}}\left(x^{2}+y^{2}\right)\right) \cos \left(2 \pi \frac{|x+y|}{\sqrt{2} \lambda}\right)$,
(10)

where $\lambda$ is a wavelength. For LM, a steering angle of $-45^{\circ}$ was also used (ie, crossed beams are superimposed). Then, the corresponding PSF is expressed by

$$
\begin{aligned}
\operatorname{PSF}(x, y)= & \exp \left(-\frac{1}{2 \sigma^{2}}\left(x^{2}+y^{2}\right)\right) \\
& \left(\cos \left(2 \pi \frac{|x+y|}{\sqrt{2} \lambda}\right)+\cos \left(2 \pi \frac{|x-y|}{\sqrt{2} \lambda}\right)\right) .
\end{aligned}
$$

The US frequency was changed from 3.5 to $12 \mathrm{MHz}$ under these conditions, and there was an US speed of $1,500 \mathrm{~m} / \mathrm{s}$, an axial sampling frequency of $30 \mathrm{MHz}$, and a beam pitch of $0.05 \mathrm{~mm}$. Considering the Nyquist theorem, if necessary, the lateral bandwidth is increased by the method described in Appendix C. By adding white noise data to the raw echo data, echo data with a SNR of $20 \mathrm{~dB}$ was also simulated.

To evaluate the measurement accuracy of a displacement, a mean and a SD vs US frequency were used. In this simulation, the axial and lateral frequencies are the same, thus for respective displacement vector measurements, the SDs of the axial displacement measurements and the corresponding lateral displacement measurements were almost the same (then, axial data obtained are not shown below).

\section{Previously developed displacement vector measurement methods - block- matching methods using local phase characteristics and other methods using instantaneous phases}

First, the accuracies of the previously developed blockmatching methods using local phase characteristics, such as 2D CSPGM, 2D AMb, and 2D DMb, and other methods 
using instantaneous phases, such as 2D AM and 2D DM, are, respectively, evaluated.

\section{D CSPGM}

Figure 4 shows the measurement accuracies of the lateral displacements measured using 2D CSPGM with LM and ASTA: conditions assuming (1) no noise in the echo data and (2) a 20-dB echo SNR are shown. The local regions used for the estimation of local cross-spectrums were 3.2 by $3.2,1.6$ by $1.6,0.8$ by 0.8 , and 0.4 by $0.4 \mathrm{~mm}$ squares.

For a rigid lateral motion, even in an ideal case with no noise, the smaller local regions used yielded smaller means (ie, biased, inaccurate means) and larger SDs (ie, unstable results), particularly, for ASTA. In the 20-dB echo SNR case (Figure $4 \mathrm{~b}$ ), almost the same means are obtained as those in the no noise case (Figure 4a). In examining the
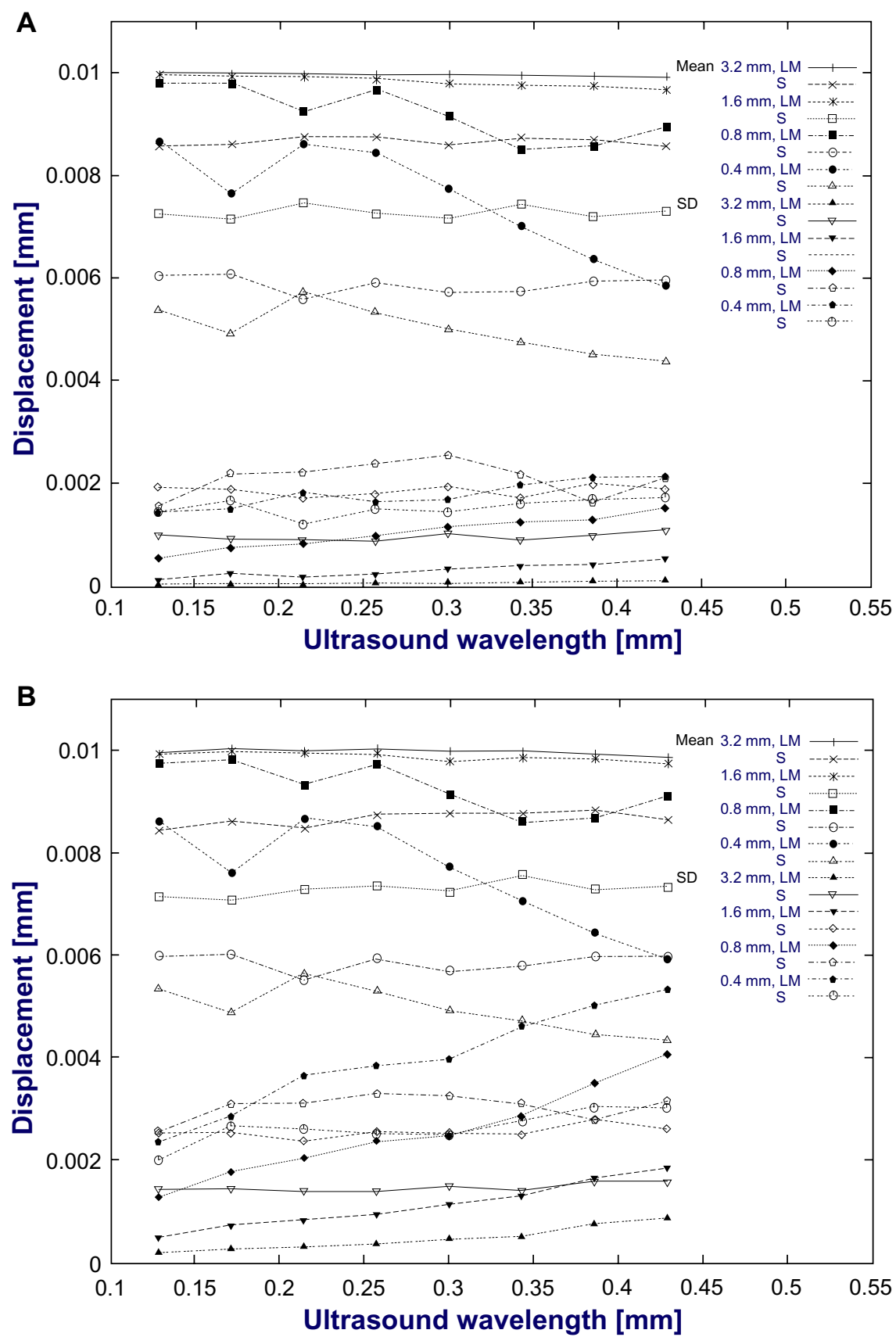

Figure 4 For lateral modulation and ASTA, means and SDs obtained for lateral displacement (0.0I mm) by 2D CSPGM. (A) The no noise echo data case and (B) a 20-dB echo SNR case. The local regions used are 3.2 by $3.2,1.6$ by $1.6,0.8$ by 0.8 , and 0.4 by $0.4 \mathrm{~mm}$ squares.

Abbreviations: SDs, standard deviations; 2D CSPGM, two-dimensional cross-spectrum phase gradient method. 
SDs, those from the 20-dB case are larger than those from the no noise case.

The comparison between ASTA and LM using the same local region size reveals that overall, ASTA yields smaller means (ie, biased ones) and larger SDs than LM. Thus, if the time required for echo data acquisition does not lead to measurement errors, LM is a more appropriate beamforming method for MCSPGM than ASTA (ie, the ranking of measurement accuracies and stabilities: LM-block $>$ S-block). This is because the simultaneous use of multiple, independent single spectra (ie, the number of independent equations) increases the measurement accuracy and stability for a least squares estimation of the displacement vector components from the gradient of the cross-spectrum phase in a frequency domain.

For instance, for ASTA, the use of local regions consisting of 3.2, 1.6, 0.8, and $0.4 \mathrm{~mm}$ squares yields small means of $0.0085,0.0075,0.0060$, and $0.0050 \mathrm{~mm}$, respectively, vs accurate means of approximately 0.0100 obtained with LM except for $0.4 \mathrm{~mm}$ square $(0.0085 \mathrm{~mm})$. Specifically, in both echo SNR cases, the means are independent of the US frequency except for LM using small local regions consisting of 0.8 and $0.4 \mathrm{~mm}$ squares, which lead to decreased means at US frequencies $<6 \mathrm{MHz}$ (at wavelengths $>0.25 \mathrm{~mm}$ ), ie, a lateral frequency of $<6 \mathrm{MHz} / \sqrt{2}$. In conjunction with this, SDs obtained with LM increase at low frequencies, particularly when using the small local regions together with the $20-\mathrm{dB}$ echo SNR. As a result, LM yields larger SDs than ASTA only for the $0.8 \mathrm{~mm}$ square at wavelengths $>0.35 \mathrm{~mm}(4.2 \mathrm{MHz})$ and $0.4 \mathrm{~mm}$ square. Thus, with low frequencies, small local regions should not be used with LM.

\section{D AM, 2D AMb, 2D DM, and 2D DMb}

Figure 5 shows measurement accuracies with lateral displacements obtained for the respective 2D AMs (2D AM and the 2D AM using block matching, ie, 2D AMb). This is shown for cases with (1) no noise in the echo data and (2) a 20-dB echo SNR. For the no noise case (Figure 5a), in addition to results obtained using the moving average of instantaneous frequencies and phase differences (referred to as only LM, ie, 2D AM) and results obtained using least squares estimations without the moving average (LM, S-block, ie, 2D AMb), results are also shown using least squares estimations with the moving average (LM, S-mvave \& block). In Figure 5b, only results obtained using LM and LM,S-block are shown. The local window/local region sizes shown are 3.2 and $0.8 \mathrm{~mm}$ squares.

With LM using the moving average, regardless of local window size, the means are accurately obtained (ie, LM and
LM,S-mvave \& block), whereas the LM,S-block method yields inaccurate means, particularly in the 20-dB echo SNR case (Figure 5b). As shown for all of the LM, LM, S-mvave \& block, and LM,S-block methods, the smaller local windows/ local regions yield larger SDs, and particularly with LM, the measurement stability degrades at low frequencies (see the SDs).

In particular, with regard to beamforming (ie, with LM and ASTA), with $2 \mathrm{D} \mathrm{AMb}$ and the local region sizes used (3.2 and $0.8 \mathrm{~mm}$ squares), the means and SDs obtained with LM are, respectively, more accurate and smaller than those obtained with ASTA, ie, the measurement accuracy and stability rankings are LM-block $>$ S-block (Figure $5 b$ ). Thus, for the block-matching methods, as with MCSPGM, LM is the appropriate beamforming method. The same reasoning can be used as with MCSPGM, ie, LM increases the number of independent equations for least squares estimations.

Regarding processing in displacement measurements, with LMs and the respective local window/local region sizes used, the size ranking of the SDs is LM-block > LM (ie, mvave) $>$ LM-mvave $\&$ block (Figure 5a). That is, from the viewpoints of measurement accuracy and stability, with LM, no block matching but a moving average should be performed. However, as expected for the measurement of a rigid motion, the combination of the moving average and block matching is the most accurate and stable for both LM and ASTA (the combination results are not shown in the following section).

With 2D DMs (ie, 2D DM and the 2D DM using block matching, ie, 2D DMb), the measurement accuracy of lateral displacements was also evaluated and similar results were obtained (then the results not shown). However, for a comparison with the accuracies of 2D CSPGM, 2D AM, and 2D $\mathrm{AMb}$, the measurement results will be shown in the following section. In summary, for the respective 2D AMs and 2D DMs, the ranking of measurement accuracy and stability is LM (mvave) $>$ LM-block $>$ S-block.

\section{Comparison of displacement vector measurement methods}

For the displacement vector measurement methods, Eq. (6) shows the ranking of measurement accuracies and stabilities.

Next, to allow comparisons of multidimensional displacement vector measurement methods for LM (ie, those using a moving average) and ASTA (using block matching), the ranking of measurement accuracy and stability is summarized in Table 1 (c, MCSPGM; a, MAM; and d, MDM). Because of 

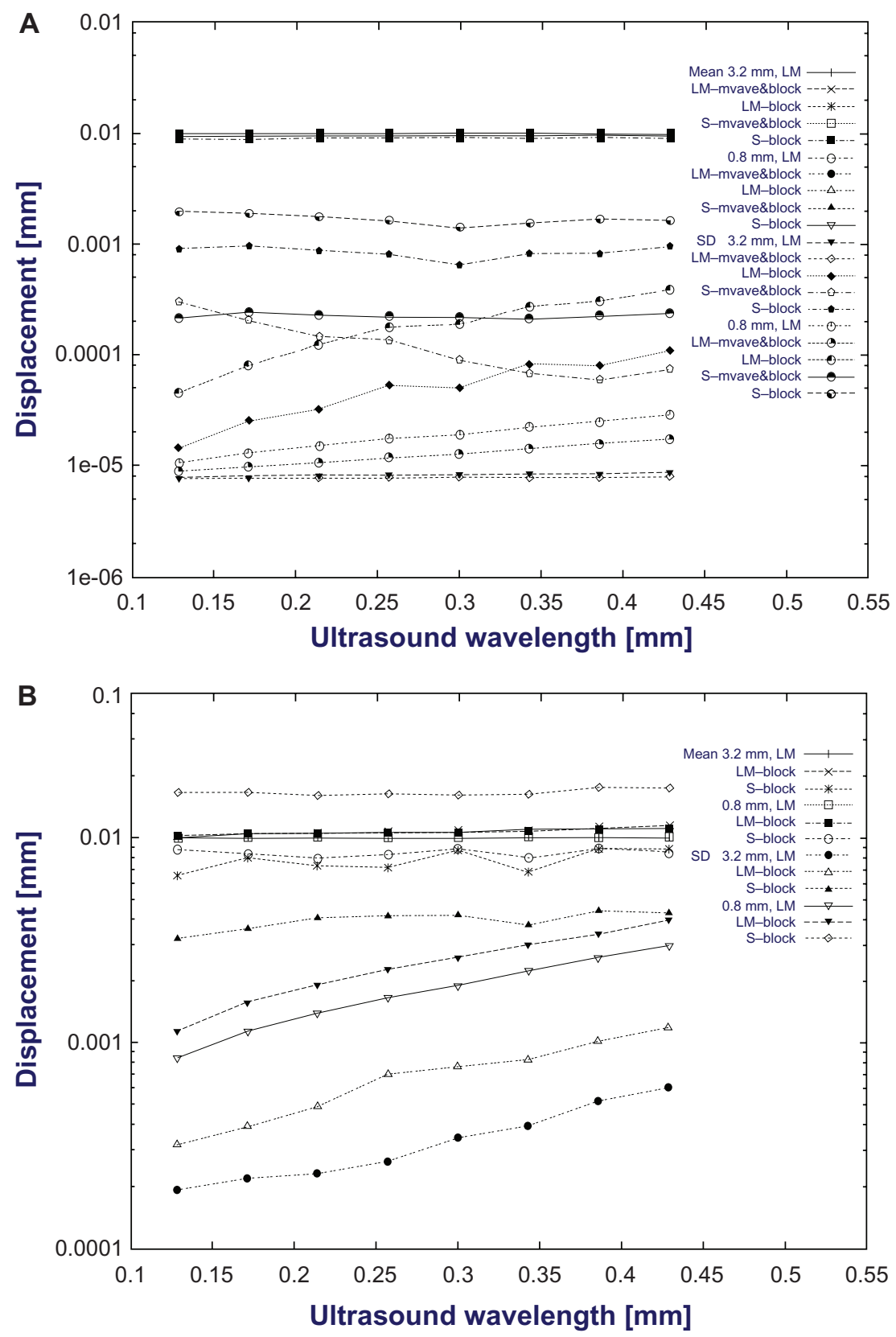

Figure 5 For lateral modulation and ASTA, means and SDs obtained for lateral displacement $(0.01 \mathrm{~mm})$ with $2 \mathrm{D}$ autocorrelation methods. (A) The no noise echo data case and (B) a 20-dB echo SNR case. In Figure 5a, in addition to those obtained using the moving average of instantaneous frequencies and phase differences (referred to as simply LM), those obtained using least squares estimations with the moving average (LM, S-mvave \& block only in Figure 5a) and without the moving averaging (LM, S-block) are also shown. In Figure 5b, only those values obtained using LM and LM, S-block are shown. The window/local region sizes shown are 3.2 and $0.8 \mathrm{~mm}$ squares.

Abbreviations: SDs, standard deviations; 2D, two dimensional; LM, lateral modulation.

Table I Ranking of accuracies and stabilities of lateral displacements

\begin{tabular}{|c|c|c|c|c|}
\hline Width, size & LM, $\infty \mathrm{dB}$ & ASTA, $\infty \mathrm{dB}$ & LM, $20 \mathrm{~dB}$ & ASTA, $20 \mathrm{~dB}$ \\
\hline 3.2 by $3.2 \mathrm{~mm}$ square & $a, c, d$ & $a, c x, d x$ & $a, d, c$ & $c x, a x, d x, x x(c, d, a x x,<7 M H z)$ \\
\hline 1.6 by $1.6 \mathrm{~mm}$ square & $a, d, c$ & $a, c x, d x$ & $a, d, c$ & $a x, x x, c x, d x, x x(c, d x x, a x x,<7 \mathrm{MHz})$ \\
\hline 0.8 by $0.8 \mathrm{~mm}$ square & a, d, cx $(<6 \mathrm{MHz})$ & $a, d x, c x(a, c, d x x)$ & a, d, cx $(<6 \mathrm{MHz})$ & $a x, c x, d x(c, a x x, d x x)$ \\
\hline 0.4 by $0.4 \mathrm{~mm}$ square & $\mathrm{a}, \mathrm{d}, \mathrm{cx}(<6 \mathrm{MHz})$ & $a, d x, c x(a, c, d x x)$ & a, d, cx $(<6 \mathrm{MHz})$ & $a x, d x, c x(c, a x x, d x x)$ \\
\hline
\end{tabular}

Note: For ASTA, in a and d, block matching is used instead of a moving average (ie, MAMb and MDMb, respectively). Biased means $(\neq 0.01 \mathrm{~mm})$ are denoted by $\mathrm{x}$, whereas SDs $(\geq 0.004 \mathrm{~mm})$ are denoted by $x \mathrm{x}$. The ranking of stabilities, which is different from that of the ranking of accuracies, is described in parentheses.

Abbreviations: a, MAM; d, MDM; c, MCSPGM; LM, lateral modulation; MAMb and MDMb, MAM and MDM using block matching; SDs, standard deviations. 
the above-mentioned bias errors with ASTA (see x in Table 1), in this report, the accuracy is evaluated by the mean, whereas the stability is evaluated by the SD, and SDs $>0.004 \mathrm{~mm}$ are denoted by xx. But for LM in Sumi, ${ }^{13}$ the accuracy was evaluated by the SNR (ie, SD/mean). For the no noise case using a local window/local region size consisting of a $3.2 \mathrm{~mm}$ square and for the $20-\mathrm{dB}$ echo SNR using a $0.8 \mathrm{~mm}$ square, the means and SDs vs US wavelength are also shown in Figures $6 \mathrm{a}$ and $6 \mathrm{~b}$, respectively.

Under the same conditions of local window/local region sizes and echo SNRs, almost the same ranking of accuracy is obtained with ASTA as with LM (Table 1). The same
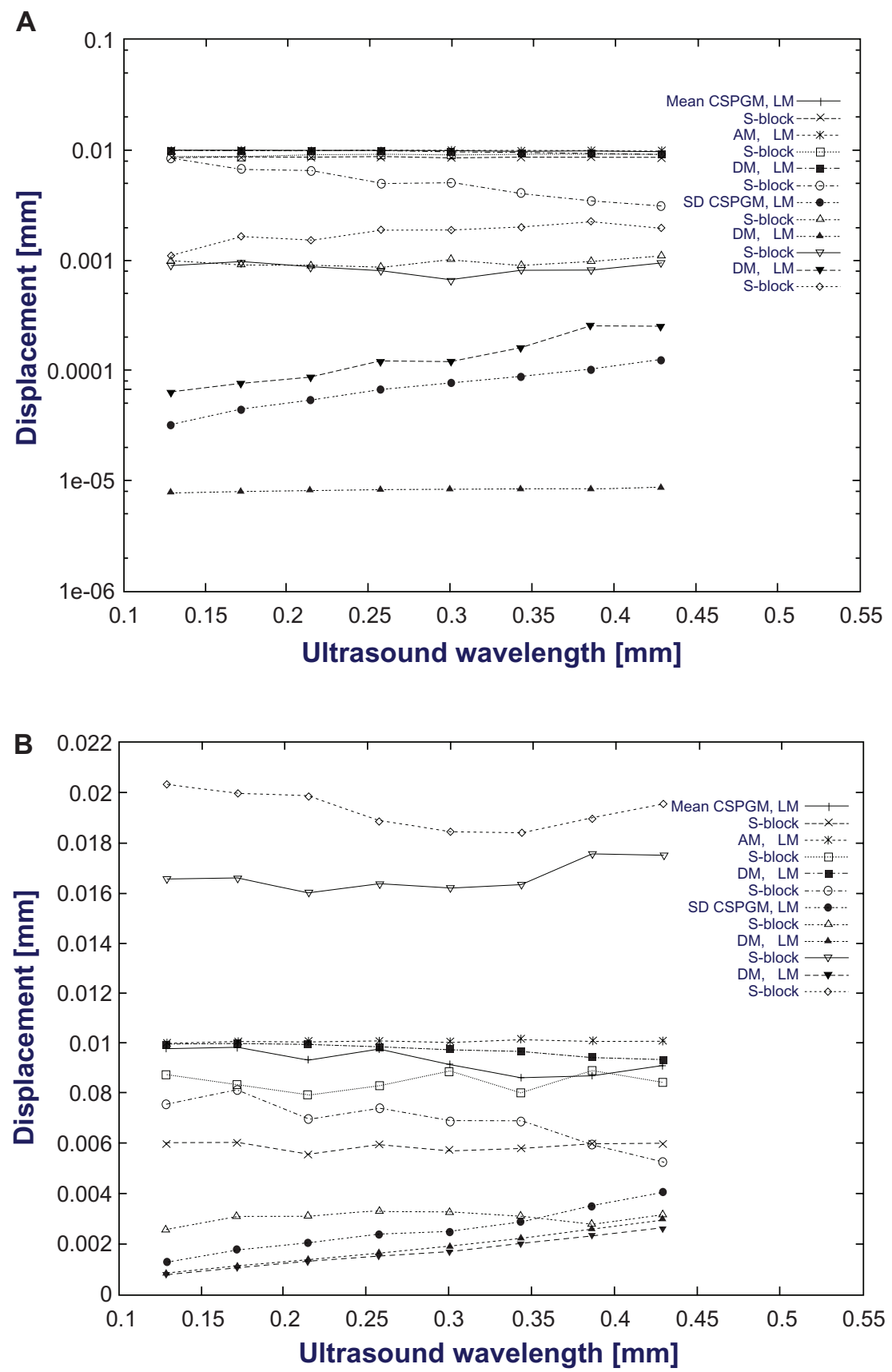

Figure 6 For LM and ASTA, means and SDs obtained for lateral displacement (0.01 mm) with 2D CSPGM, AM, AMb, DM, and DMb (ie, using a moving average or block matching). Shown are (A) the no noise echo data case using a local window/region size consisting of a $3.2 \mathrm{~mm}$ square and (B) a $20 \mathrm{~dB}$ echo SNR using a $0.8 \mathrm{~mm}$ square. Abbreviations: LM, lateral modulation; SDs, standard deviations; 2D CSPGM, two-dimensional cross-spectrum phase gradient method; AM, autocorrelation method; AMb, autocorrelation method using a block-matching; DM, Doppler method; DMb, Doppler method using a block-matching; SNR, signal-to-noise ratio. 
ranking can also be confirmed as with LM and obtained with simulations of a displacement vector $(0.01$ and $0.01 \mathrm{~mm})$ measurement (ie, table IV in Sumi ${ }^{13}$ ). That is, for a high echo SNR, the order of measurement accuracy is a, c, d, and as the echo SNR decreases from $20 \mathrm{~dB}$ or as the local window/ local region size decreases from a $3.2 \mathrm{~mm}$ square, the order changes are as follows: adc, dac, and dca $(0.7 \mathrm{~dB}$ or $0.4 \mathrm{~mm}$ square). That is, as with LM, both the accuracy and stability of the measurement methods are dependent on the echo SNR and the local window/local region size used.

For ASTA in the no noise case, local window/local region sizes of 3.2 (Figure $6 \mathrm{a}$ ) and $0.8 \mathrm{~mm}$ squares (figure not shown) respectively yielded large SDs from 0.0008 to 0.0020 (a $<$ $\mathrm{c}<\mathrm{d}$ ) and 0.0013 to $0.0043 \mathrm{~mm}$ ( $\mathrm{a}<\mathrm{c}<\mathrm{dxx}$ ). With regard to the means, with an increasing US frequency, the value of $\mathrm{dx}$ for the $3.2 \mathrm{~mm}$ square decreases to $0.003 \mathrm{~mm}$ (an inaccurate level), the value of $\mathrm{dx}$ for the $0.8 \mathrm{~mm}$ square decreases to $0.007 \mathrm{~mm}$ (figure not shown), and the value of cx for the $0.8 \mathrm{~mm}$ square remains at $0.006 \mathrm{~mm}$ (ie, biased values, figure not shown).

For ASTA with a 20-dB echo SNR, when frequencies are lower than $7 \mathrm{MHz}$ (wavelengths $\geq 0.214 \mathrm{~mm}$ ), even the use of a large local region size consisting of a $3.2 \mathrm{~mm}$ square yields large SDs, ie, 0.0013-0.0042 mm (c < a $<\mathrm{dxx}$, figure not shown); and the use of a small local region size consisting of a $0.8 \mathrm{~mm}$ square yields $\mathrm{SDs}>0.016$ and $0.018 \mathrm{~mm}$ for axx and dxx, respectively, and a SD of about $0.003 \mathrm{~mm}$ for $\mathrm{c}$ as shown in Figure 6b. Similarly, with regard to the means, with an increasing US frequency for the $3.2 \mathrm{~mm}$ square, the value of $\mathrm{dx}$ decreases from 0.0070 to $0.0033 \mathrm{~mm}$, and the values of ax and cx remain at about 0.0080 and $0.0083 \mathrm{~mm}$, respectively (figure not shown). For the $0.8 \mathrm{~mm}$ square, the value of $\mathrm{dx}$ decreases from 0.0080 to $0.0050 \mathrm{~mm}$, and the value of ax and cx for the $0.8 \mathrm{~mm}$ square remains at about 0.0080 and $0.0060 \mathrm{~mm}$ (ie, also biased values).

In summary, for the 20-dB echo SNR, although MDM yields small means $(\approx 0.003 \mathrm{~mm})$ at low frequencies, MAM and MCSPGM yield rather accurate means for the US frequencies. Moreover, MAM and MDM yield very unstable measurements (SDs $>0.004 \mathrm{~mm}$ ). Thus, for practical applications of block matching for ASTA, MCSPGM will be the most useful if a phase matching is performed, whereas for LM, all multidimensional methods are useful, although they should be properly selected according to the echo SNR, the required spatial resolution, and the required calculation speed. ${ }^{13}$ Recall that in this simulation for LM, bias errors occur for cx with a local window/region size smaller than a $0.8 \mathrm{~mm}$ square and an US frequency $<6 \mathrm{MHz}$ (wavelengths $\geq 0.25 \mathrm{~mm}$ ).

Next, for ASTA, lateral displacement measurements were tested for new 2D AM and 2D DM using a mirror setting (ie, no block matching) for the steered beams with a defined steering angle.

\section{New lateral displacement measurement methods - 2D AM and 2D DM using mirror settings}

Figure 7 shows the measurement accuracy of a lateral displacement, which was obtained with the 2D AM using a mirror setting with a steered beam for ASTA. The accuracy obtained for LM is also shown (Figure 7). Because the use of a moving average did not generate a bias error, the means obtained were not shown. The local windows used for the estimation of the moving averages of the instantaneous phase differences and instantaneous frequencies were 3.2, 1.6, 0.8, and $0.4 \mathrm{~mm}$ squares.

With the local windows used, in the no noise case (Figure 7a), there is no difference between the SD values for LM and ASTA. The use of small windows yields unstable results. However, interestingly, SDs for LM are larger than those for ASTA in the 20-dB case (Figure 7b). That is, for a lateral displacement measurement using MAM and MDM, ASTA is an appropriate beamforming method. Thus, for lateral displacement measurements using MAM, the mirror setting is useful, and the ranking of measurement accuracy and stability is shown in Eq. (7). Also note that for the mirror setting, with a decreasing US frequency, SDs become larger. Because similar results are also obtained with the DM using a mirror setting, they are not shown in this subsection. However, for a comparison with the 1D method, the measurement results will be shown in the end of this section.

\section{ID methods for measurements of displacement vector and lateral displacement}

Figure 8 , for the 20-dB echo SNR case, shows the SD vs US frequency obtained with 1D AM. LM and ASTA were used, respectively. In the no noise case, there were no differences between LM and ASTA, and those results were not shown. For the moving averages of the instantaneous phase differences and instantaneous frequencies, local windows consisting of $3.2,1.6,0.8$, and $0.4 \mathrm{~mm}$ squares were used. 

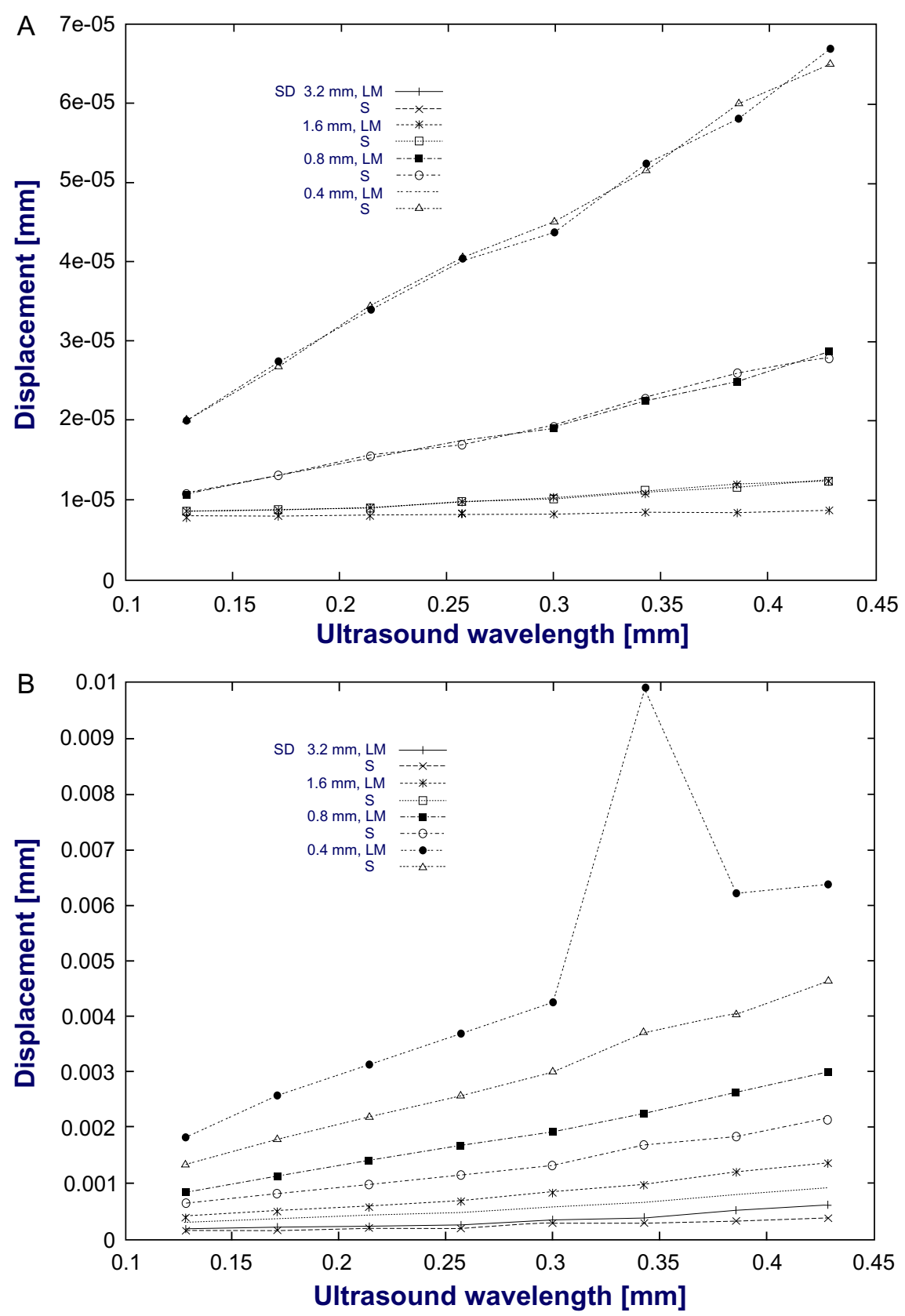

Figure 7 For LM and ASTA, SDs obtained for lateral displacement $(0.01 \mathrm{~mm})$ with 2D AM (A) in a no noise echo data case and (B) in a 20-dB echo SNR case. For LM, a moving average is used; for ASTA, a moving average and a mirror setting are used. The local windows used are 3.2, I.6, 0.8, and $0.4 \mathrm{~mm}$ squares. Abbreviations: LM, lateral modulation; SDs, standard deviations; 2D AM, two-dimensional autocorrelation method; SNR, signal-to-noise ratio.

For LM, "BOTH" indicates the case when both independent single spectra were used for the calculation of $c / f y$; "CONJ PRODUCT" is the case where the newly developed demodulation method was used for both spectra (Appendix A); and "DEMODU" is the case where the demodulation method described by Anderson ${ }^{26}$ was used for the 2 spectra.

As shown for all of the local windows used, ASTA yields smaller SDs than LM, ie, Eq. 9. With LM, the size ranking of the SDs is DEMODU $>$ BOTH $>$ CONJ PRODUCT.
The differences between them are more notable when the smaller local window is used. Thus, the newly developed CONJ PRODUCT is the most useful demodulation method of those discussed in this report. As with the 2D methods, with a decreasing US frequency, the SDs become larger. For the corresponding 1D DMs, the results obtained were similar to those obtained with 1D AMs (not shown). However, a comparison with the 2D method will be shown in the following section. 


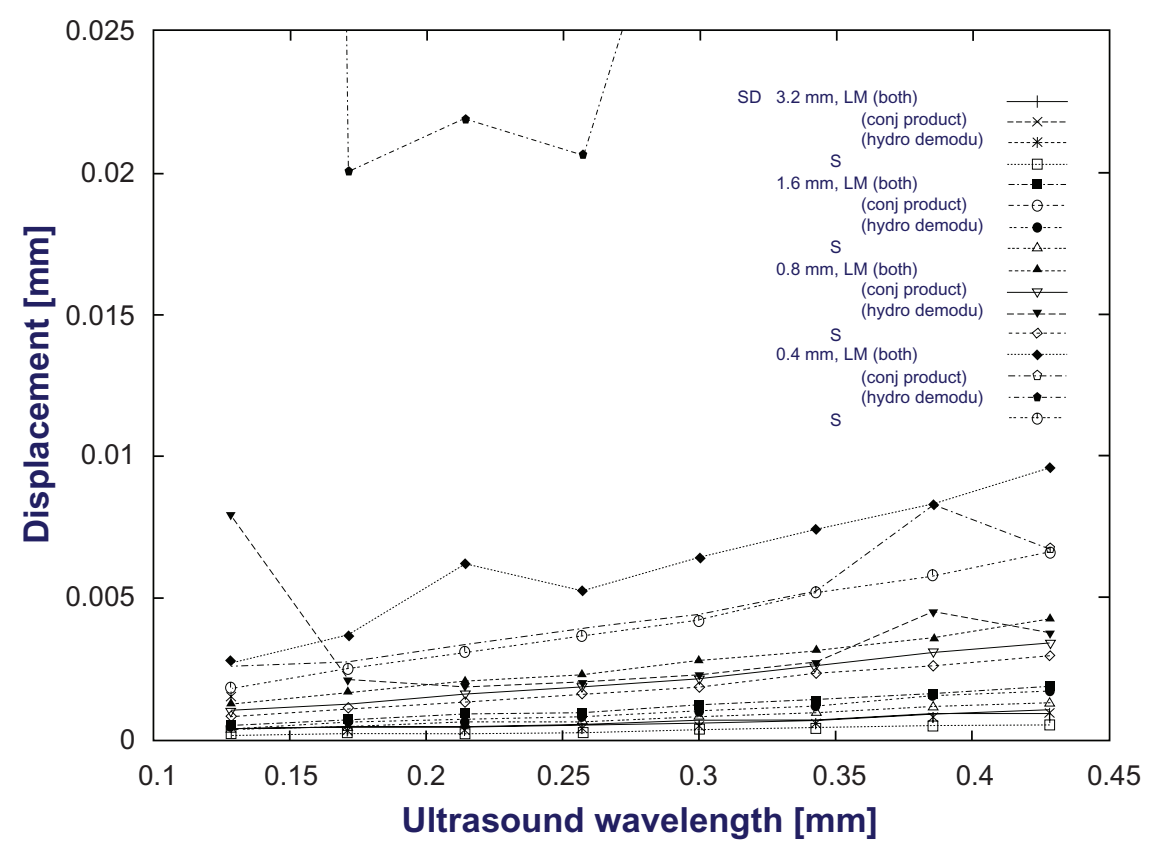

Figure 8 For LM and ASTA, SDs obtained for lateral displacement $(0.01 \mathrm{~mm})$ with ID AM in a 20-dB echo SNR case. The local windows used were 3.2, I.6, 0.8, and $0.4 \mathrm{~mm}$ squares. "BOTH" indicates that both independent single spectra were used for the calculation of $c / f y$, "CONJ PRODUCT" indicates that the recently developed demodulation method was used for both spectra (Appendix A), and "DEMODU" indicates that the demodulation method described in Anderson et al ${ }^{26}$ was used for the 2 spectra, respectively.

Abbreviations: LM, lateral modulation; SDs, standard deviations; ID AM, one-dimensional autocorrelation method; SNR, signal-to-noise ratio.

\section{Comparisons of 2D and ID methods for measurements of displacement vector and lateral displacement}

Figure 9 shows the results for LM and ASTA with a 20-dB echo SNR and shows the SD vs the US frequency obtained with 2D CSPGM, 2D AM, and 2D DM using a moving average for LM and a mirror setting for ASTA (ie, no block matching), respectively, 1D AM, and 1D DM for local window/local regions consisting of (1) 3.2 and (2) $0.8 \mathrm{~mm}$ squares. Because MAM and MDM using block matching yield inaccurate and unstable measurements, these results were not provided in this subsection (Table 1; it should be recalled that they have SDs $>0.016 \mathrm{~mm}$ as shown at the beginning of this section).

For the methods except for 2D CSPGM (ie, block matching), ASTA yields more stable and accurate lateral displacement measurements than LM as already shown. Under the same conditions, as shown in Sumi, ${ }^{13}$ with LM the measurement accuracy and stability of the 2D methods (2D AM and DM) are, respectively, higher than those of the corresponding 1D methods (1D AM and DM). Interestingly, the same relations can also be confirmed for ASTA. With regard to the SDs, when using the $0.8 \mathrm{~mm}$ square (Figure $9 \mathrm{~b}$ ) for the AMs, they were $0.00065-0.00215 \mathrm{~mm}$ vs $0.00085-$ $0.00300 \mathrm{~mm}$; for the DMs, they were $0.00065-0.00200 \mathrm{~mm}$ vs $0.00085-0.00275 \mathrm{~mm}$. That is, for ASTA, 2D methods also yield more stable and accurate lateral displacement measurements than the corresponding 1D methods. However, the 1D methods using CONJ PRODUCT are still useful because the number of required calculations is lower than that required for the multidimensional methods.

\section{Discussions and conclusions}

A new beamforming method is proposed in this report, which is simpler than LM for measuring a lateral displacement and/ or a displacement vector; this method uses the steering of beams with a defined steering angle (ASTA) for an arbitrary orthogonal coordinate system. For ASTA, the MCSPGM, the MAM, and the MDM, using block matching (ie, MAMb and MDMb), and a MCCM (not shown in this report) can be used for measurements of a displacement vector and a lateral displacement (ie, block-matching methods), whereas the MAM and the MDM, using a moving average and a mirror setting, and the 1D methods can be used only for a lateral displacement measurement (Figure 1).

To increase the measurement accuracy of a lateral displacement component, as for LM, the steering angle used is as large as possible. For a lateral displacement measurement (eg, blood flow in a carotid artery), a lateral coordinate must correspond to the direction of the target lateral motion. However, for a displacement vector measurement to describe a complex tissue motion (eg, cardiac motion), if the axial 

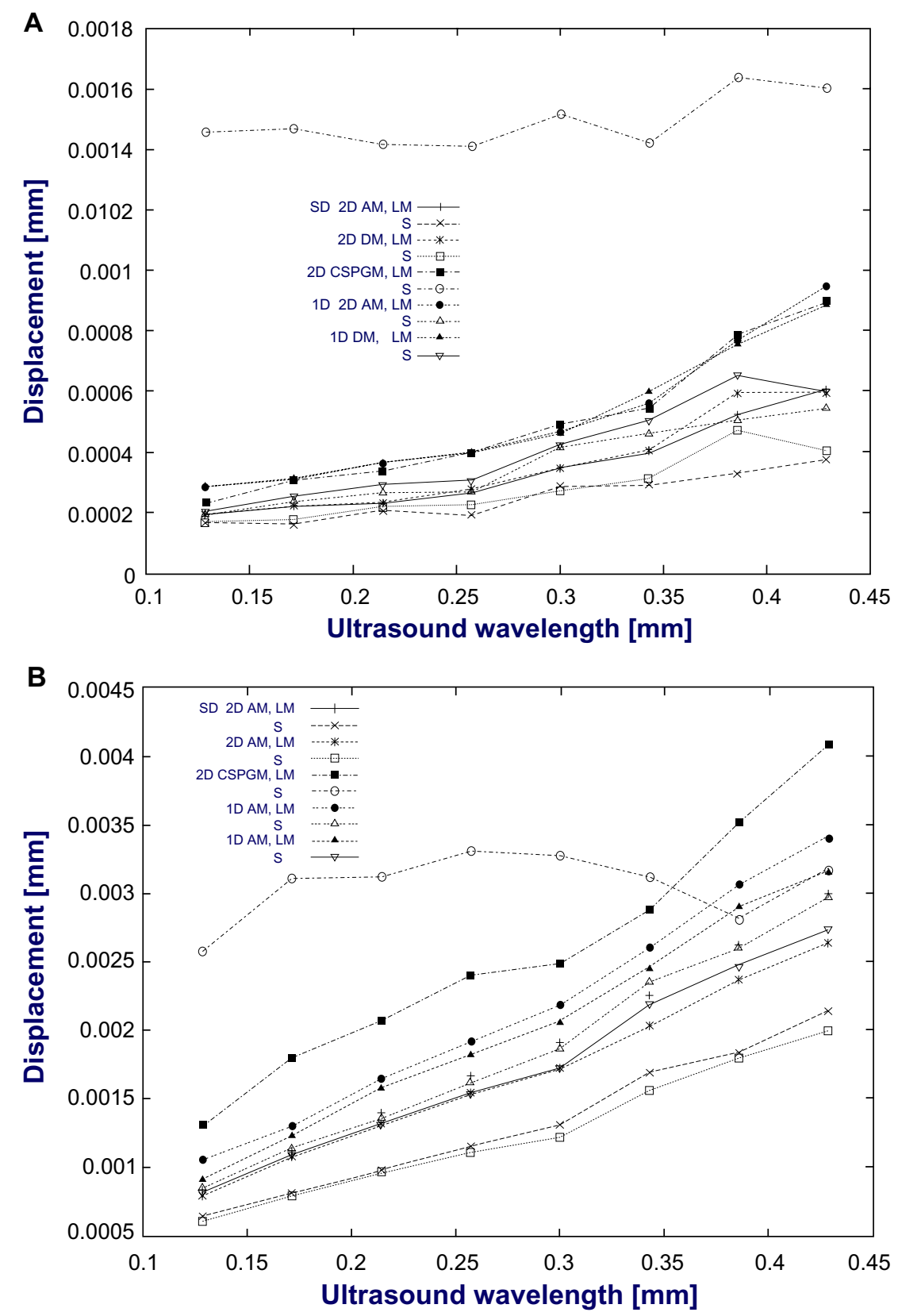

Figure 9 For LM and ASTA, SDs were obtained for lateral displacement $(0.01 \mathrm{~mm})$ with 2D AM and 2D DM using only a moving average (LM) and a mirror setting (ASTA), 2D CSPGM, and ID AM and ID DM using LM with CONJ PRODUCT (Appendix A) and ASTA in a $20 \mathrm{~dB}$ echo SNR case (A) using 3.2 and (B) $0.8 \mathrm{~mm}$ squares.

Abbreviations: LM, lateral modulation; SDs, standard deviations; 2D, two dimensional; AM, autocorrelation method; DM, Doppler method; CSPGM, cross-spectrum phase gradient method; ID, one dimensional; SNR, signal-to-noise ratio.

coordinate corresponds to the depth in a target tissue and the steering angle is $45^{\circ}$, then the same measurement accuracy can be obtained for the axial and lateral displacement measurements. However, for practical applications, the echo SNR from a steered beam must also be considered as for LM. That is, an overly large steered angle will make the echo SNR low.

For measurements of a displacement vector and a lateral displacement using LM, in addition to MCSPGM, MAM, and MDM using a moving average, a combination of $1 \mathrm{D}$ methods and the newly developed demodulation method (Appendix A: CONJ PRODUCT) can be used. As with LM or no LM using the phase matching, ${ }^{9-13}$ the useful displacement measurement methods can be properly combined for measurements of a displacement vector and a lateral displacement with ASTA, ie, MCCM, MCSPGM, MAMb, MDMb, MAM, MDM, and such 1D methods.

In addition, comparisons between LM and ASTA were performed primarily with concern about the generation of artifacts due to the difference in echo data acquisition times in the end of the second section. For LM, problems 
1-5 that decrease measurement accuracy were considered, although problems 1 and 5 will be managed by the newly developed virtual source (Appendix B). In contrast, with ASTA, the number of available methods is limited to obtain displacement vector measurements as summarized above. However, only for lateral displacements, measurements can be performed absolutely. Moreover, comparably none of the concerns $1-5$ listed above will be a problem, and a simple beamforming can increase the reliability of realtime measurements together with accuracy in displacement measurements. ASTA is also robust to the inhomogeneity of tissue properties, such as a propagation speed. As mentioned briefly in the second section, a proposed mirror setting of the spectra will also be applied to a quasi-LM imaging together with the lateral displacement measurement using MAM and MDM.

With 2D simulations, except for the difference in echo acquisition times, the measurement accuracy and stability of lateral displacements were determined (Figure 1). It was confirmed that LM is a more appropriate beamforming method for block-matching methods (ie, all MCSPGM, MAMb, $\mathrm{MDMb}$, and MCCM are useful), and ASTA is appropriate for MAM and MDM using a mirror setting and 1D methods. However, if a displacement vector measurement must be performed with ASTA, MCSPGM is the most useful with the combination with a phase matching. For a displacement vector measurement or a lateral displacement measurement using multidimensional methods, the ranking for measurement accuracy and stability are mirror setting (ASTA) $>$ LMmvave $>$ LM-block $>$ S-block. Although block matching requires fewer calculations than a moving average, a lower accuracy is obtained. As with LM, multidimensional methods yield more accurate measurements with ASTA than do the corresponding 1D methods. Moreover, being dependent on the echo SNR and the required spatial resolution (the local window/local region size) and calculation speed, as with LM, a proper displacement measurement method should also be selected for ASTA.

In addition to measurements of blood flow and tissue elasticity (displacement, strain, shear modulus, etc) for diagnosis, displacement measurements using ASTA can also be applied to confirming tissue regeneration through elasticity changes by using low-invasive coagulation therapies with high-intensity focused ultrasound, rf, or micro-electromagnetic waves ${ }^{34}$ and in the planning of such treatments with temperature (thermal strain) measurements and reconstructions of tissue thermal properties. ${ }^{41}$ Such treatments degrade the echo SNR at the locus of the displacement measurement. Because every tis- sue also has its own motion (heart, liver, etc) and, occasionally, there are obstacles, such as the presence of bones, the target tissue will determine the selection of the proper beamforming method with a choice between LM and ASTA together with the selection of the proper displacement measurement method. ASTA that is realized by the combination of the non-steering and the rotation of a coordinate system is effective, particularly when a high echo SNR is required or obstacles exist (see Appendix C) ${ }^{42}$ For LM, this rotation also allows the use of nonsymmetric steering angles with $1 \mathrm{D}$ displacement measurement methods. ${ }^{42}$ ASTA will open up new aspects of displacement measurements together with LM. In this report, the actual anisotropic geometry of a steered beam (ie, acoustical pressure) is not dealt with. Thus, future studies will be performed primarily using in vivo experiments. Previously developed optimization methods for determining beamforming parameters, such as an apodization function involving delays ${ }^{20,21}$ will be used for ASTA and LM. Moreover, for a new displacement vector measurement using own developed methods, the division of spectra in a frequency domain will be reported elsewhere. ${ }^{42}$ From a single spectra, plural analytic signals (spectra) can be obtained. To increase measurement accuracy, low frequency spectra can also be disregarded.

\section{Disclosure}

The author reports no conflicts of interest in this work.

\section{References}

1. Satomura S. Study of the flow patterns in peripheral arteries by ultrasonics. J Acoust Soc Jpn. 1959;15:151-158.

2. Barker DW. Pulsed ultrasonic Doppler blood-flow sensing. IEEE Trans Sonics Ultrason. 1970;17(3):170-185.

3. Kasai C, Namekawa K, Koyano A, Omoto R. Real-time two-dimensional blood flow imaging using an autocorrelation technique. IEEE Trans Sonics Ultrason. 1985;32:458-464.

4. Foster SG, Embree PM, O’Brien WD Jr. Flow velocity profile via timedomain correlation: Error analysis and computer simulation. IEEE Trans Ultrason Ferroelectr Freq Contr. 1990;37:164-175.

5. Wilson LS, Robinson DE. Ultrasonic measurement of small displacements and deformations of tissue. Ultrason Imag. 1982;4:71-82.

6. Ophir J, Cespedes I, Ponnekanti H, Yazdi Y, Li X. Elastography: a quantitative method for measuring the elasticity of biological tissues. Ultrason Imag. 1991;13:111-134.

7. Trahey GE, Allison JW, von Ramm OT. Angle independent ultrasonic detection of blood flow. IEEE Trans Biomed Eng. 1987;34: 965-967.

8. Yagi S, Nakayama K. Local displacement analysis of inhomogeneous soft tissue by spatial correlation of rf echo signals. Proceedings of World Federation of Ultrasound in Medicine and Biology. Meeting; 1988 Oct; Washington DC, US. p. 113.

9. Sumi C, Suzuki A, Nakayama K. Phantom experiment on estimation of shear modulus distribution in soft tissue from ultrasonic measurement of displacement vector field. IEICE Trans Fundamental. 1995;78A: 1655-1664.

10. Sumi C. Fine elasticity imaging on utilizing the iterative rf-echo phase matching method. IEEE Trans Ultrason Ferroelectr Freq Contr. 1999; 46:158-166. 
11. Sumi C. Digital measurement method of tissue displacement vector from instantaneous phase of ultrasonic echo signal. Technical report of Japan Society of Ultrasound Medicine; 2002 Dec; Tokyo, Japan. p. $37-40$.

12. Sumi C. Multidimensional displacement vector measurement methods utilizing instantaneous phase [CD-ROM]. Proceedings of the 27th International Conference IEEE Engineering in Medicine and Biology Society; 2005 Sep; Shanghai, China.

13. Sumi C. Displacement vector measurement using instantaneous ultrasound signal phase - multidimensional autocorrelation and Doppler methods. IEEE Trans Ultrason Ferroelectr Freq Contr. 2008;55: 24-43.

14. Chen X, Zohdy MJ, Emelianov SY, O’Donnell M. Lateral speckle tracking using synthetic lateral phase. IEEE Trans Ultrason Ferroelectr Freq Contr. 2004;51:540-550.

15. Sumi C, Ebisawa T. Phantom experiments of axial strain measurements using multidimensional autocorrelation method, multidimensional Doppler method and direct strain measurement method. Acoust Sci Tech. 2009;30:124-131.

16. Sumi C. Ultrasonic axial strain measurement for lateral tissue deformation. Ultrasound Med Biol. 2007;33:1830-1837.

17. Sumi C. Improvement of measurement accuracy of displacement vector by lateral modulation [in Japanese]. Proceedings of the 2004 Autumn Meeting Acoustical Society of Japan; 2004 Sep; Okinawa, Japan; p. 1353-1354.

18. Sumi C, Noro T, Tanuma A. Effective lateral modulations with applications to shear modulus reconstruction using displacement vector measurement. IEEE Trans on Ultrason Ferroelect Freq Contr. 2008;55: 2607-2625.

19. Sumi C, Tanuma A. Comparison of parabolic and Gaussian lateral cosine modulations in ultrasound imaging, displacement vector measurement, and elasticity measurement. Jpn J Appl Phys. 2008;47(5B): 4137-4144.

20. Sumi C, Komiya Y, Uga S. A demonstration of optimal apodization determination for proper lateral modulation. Jpn J Appl Phys. 2009; 48(7B):07GJ06.

21. Sumi C. Determination of lateral modulation apodization function using regularized, weighted least squares estimation. Int J Biomed Imaging 2010;2010:635294.

22. Steinberg BD. Principles of Aperture and Array System Design. New York: Wiley; 1976.

23. Jensen JA. A new method for estimation of velocity vectors. IEEE Trans Ultrason Ferroelectr Freq Contr. 1998;45:837-851.

24. Anderson ME. Multi-dimensional velocity estimation with ultrasound using spatial quadrature. IEEE Trans Ultrason Ferroelectr Freq Contr. 1998;45:852-861.

25. Jensen JA. A new estimator for vector velocity estimation. IEEE Trans Ultrason Ferroelectr Freq Contr. 2001;48:886-894.

26. Anderson ME. A heterodyning demodulation technique for spatial quadrature. Proceeding of the 2000 IEEE Ultrasonics Symposium; 2000 Oct; San-Juan, Puerto Rico. p. 1487-1490.

27. Liebgott H, Fromageau J, Wilhjelm JE, Vray D, Delachartre P. Beamforming scheme for 2-D displacement estimation in ultrasound imaging EURASIP J Appl Signal Processing. 2005;8:1212-1220.

28. Sumi C, Suzuki A, Nakayama K. Estimation of shear modulus distribution in soft tissue from strain distribution. IEEE Trans Biomed Eng. 1995;42:193-202.

29. Sumi C. Increasing accuracy of tissue shear modulus reconstruction using ultrasonic strain tensor measurement - regularization and lateral modulation. Acoust Imag. 2008;29:59-69.

30. Sumi C, Shimizu K, Matsui N. Proper analytic point spread function for lateral modulation. Jpn J Appl Phys. 2010; 48(7B) (in press).
31. Fox MD. Multiple crossed-beam ultrasound Doppler velocimetry. IEEE Trans Ultrason Ferroelectr Freq Contr. 1978;25:281-286.

32. Techavipoo U, Chen Q, Varghese T, Zagzebski JA. Estimation of displacement vectors and strain tensors in elastography using angular isonifications. IEEE Trans Med Imaging. 2004;23: 1479-1489.

33. Sumi C. Displacement measurement method and apparatus, strain measurement method and apparatus, elasticity and viscoelasticity constants measurement apparatus, and the elasticity and viscoelasticity constants measurement apparatus based treatmemt apparatus. Japanese Patent 4260523, Apr 30, 2009 (application Apr 25, 2002); US Patent (allowed, but, number not assigned yet, application 10/326,526, Dec 23, 2002).

34. Sumi C. Usefulness of ultrasonic strain measurement-based shear modulus reconstruction for diagnosis and thermal treatment. IEEE Trans Ultrason Ferroelectr Freq Contr. 2005;52:1670-1689.

35. Tanter M, Bercoff J, Sandrin L, Fink M. Ultrafast compound imaging for 2-D motion vector estimation: application to transient Elastography. IEEE Trans Ultrason Ferroelectr Freq Contr. 2002;49: 1363-1374.

36. Sumi C, Suzuki A, Nakayama K, Kubota M. Estimation of stiffness distribution in soft tissue from displacement vector measurement [in Japanese]. Jpn J Med Ultrasonics. 1995;22 Suppl 1:44.

37. Konafagou E, Ophir J. A new elastographic method for estimation and imaging of lateral displacement, lateral strains, corrected axial strains and Poisson's ratios in tissues. Ultrasound Med Biol. 1998;24: 1183-1199.

38. Sumi C, Uga S, Komiya Y. Lateral modulation - use of virtual source (11th report) [in Japanese]. Proceedings of the 2009 Spring Meet Acoustical Society of Japan; 2009 Mar; Tokyo, Japan; p. 1381-1382.

39. Sumi C, Matsui N, Shimizu K, Takanashi Y. Preliminary experiments on virtual source for lateral modulation. Proceeding of the 8th International Conference on the Ultrasonic Measurement and Imaging of Tissue Elasticity; 2009 Sep 14-17; Vlissingen, Netherlands. http:// www.elasticityconference.org/prior_conf/2009/PDF/088SumiC02FP. pdf; 2010

40. Sumi C, Shimizu K, Matsui N. Applications of ultrasonic beam steering to tissue imaging [in Japanese]. IEICE Technical Report. US2009-28, 2009 Jul:33-37.

41. Sumi C, Yanagimura H. Reconstruction of thermal property distributions of tissue phantoms from temperature measurements - thermal conductivity, thermal capacity and thermal diffusivity. Phys Med Biol. 2007;52:2845-2864.

42. Sumi C. Displacement measurement method and apparatus, and ultrasound diagnostic apparatus. Japanese Patent Application 2009-209656, Sep 10, 2009; US Patent Application 12/833, 072, July 9, 2010.

43. O'Donnell M, Thomas LJ. Efficient synthetic aperture imaging from a circular aperture with possible application to catheter-based imaging. IEEE Trans Ultrason Ferroelectr Freq Contr. 1992;39: 366-380.

44. Karaman M, Li P-C, O’Donnell M. Synthetic aperture imaging for small scale systems. IEEE Trans Ultrason Ferroelectr Freq Contr. 1995;42: $429-442$.

45. Lockwood GR, Talman JR, Brunke SS. Real-time 3-D ultrasound imaging using sparse synthetic aperture beamforming. IEEE Trans on Ultrason Ferroelect Freq Contr. 1998;45:980-988.

46. Oddershede N, Jensen JA. Effects influencing focusing in synthetic aperture vector flow imaging. IEEE Trans Ultrason Ferroelectr Freq Contr. 2007;54:1811-1825. 


\section{Appendices}

Appendix A:A new demodulation method for displacement vector measurement using LM and ID measurement methods

When LM is performed, a demodulation method ${ }^{25,26}$ can be used together with a 1D measurement method. However, a new demodulation method has been developed (see "CONJ PRODUCT" used in simulations, ie, third section). For example, when a 2D displacement vector $(d x, d y)$ is measured, after obtaining instantaneous phase differences between premotion and postmotion in exponential form, eg, expj $(f x d x+f y d y)$ and $\exp j(f x d x-f y d y)$, by calculating their product and their conjugate product, $\exp j(2 f x d x)$ and $\exp j(2 f y d y)$, are obtained. Thus, by dividing their phase differences by the respective frequencies $2 f x$ and $2 f y$ in the 1D measurement methods, the displacement vector $(d x, d y)$ can be obtained. Note that because 2-fold instantaneous frequencies are used, large bandwidths should be used in advance (Nyquist theorem) or an increase in bandwidth (interpolation) must be performed as described in Appendix C. This method can be extended simply to a 3D displacement vector measurement, in which the 2-fold instantaneous frequencies appears similarly after the handling of 3 or 4 instantaneous phase differences between premotion and postmotion in exponential form.

\section{Appendix B:A virtual source for obtaining ultrasound intensity}

When dealing with deeply situated tissues, LM requires the use of a large physical aperture. ${ }^{13}$ This limits the applications of LM. To overcome this limitation, the use of a new virtual source is proposed. ${ }^{38,39}$ With this method, regardless of the focus position of an individual element aperture, proper scattering is used to provide virtual sources. For instance, by setting virtual sources in tissues, an arbitrary field of vision (FOV) can be obtained regardless of the aperture geometry (convex, linear, etc). A proper random scattering medium can also be installed in the transducer ${ }^{18}$ or between the transducer and a target body. If the scatters work as like point acoustical sources, a lateral resolution can also be improved for the echo imaging and various measurements. In addition, virtual sources (or receivers) will also be realized in null spaces along or beside the short physical array apertures by searching for the corresponding echo data in the acquired echo data set.

By setting virtual sources behind the aperture as shown in Appendix 1 and by using decay weights (ie, values obtained from the decay of a propagating wave, such as a spherical wave generated by a point virtual source), a larger

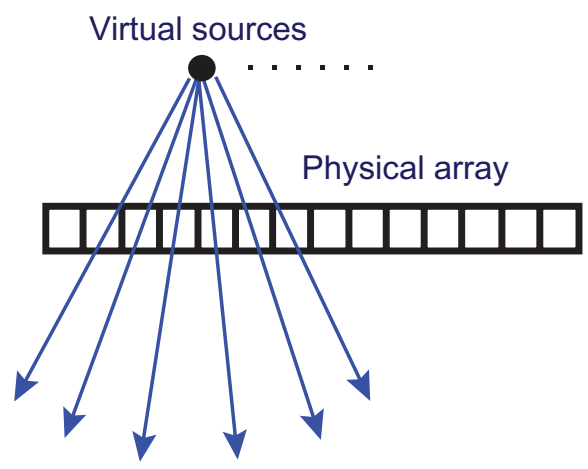

Appendix I New virtual source for gaining ultrasound intensity; eg, when using a linear array.

intensity ultrasound signal can be obtained than from classical SAs. ${ }^{38,39,43-46}$ A large FOV can also be obtained.

Other virtual sources different from a point source can also be used: for instance, a source having a finite aperture. Decay weights can be calculated analytically or numerically. Regarding the scattering angle vs the US penetration, the geometry and acoustical impedance of scatter and media, and some regular, irregular, or random alignment or distribution of scatters are also examined in conjunction with a physical source. That is, a realization of the proper diffraction is a research target. Our previously developed optimization method $^{20,21}$ for determining beamforming parameters, such as an apodization function involving delays, is also used to determine the position, geometry, and intensity of virtual sources. Preliminary experimental results are presented in Sumi. ${ }^{38,39}$ The point virtual sources should be set in the neighborhood of the physical sources that are being used.

\section{Appendix C: Increase in lateral frequency through an increase in the steering angle}

For LM and ASTA, to achieve a high lateral frequency, it is necessary to increase the beam steering angle. However, when the pitch of the element or beam is too large, an aliasing of spectra occurs (Appendix 2a). To mitigate the occurrence of aliasing, the beam pitch is kept appropriately small. For LM using the superposition of multiple steered beams canalsics be ASTA, the respective beams can also be interpolated in a frequency domain by increasing a lateral bandwidth padded by zeros (Appendix 2b).

In conjunction with this, the separation of overlapped beams in a spatial domain, such as crossed beams (LM, Figure 2c), grating lobes, side lobes (Appendix 3), and signals received from some other sources, can be separated in a frequency domain. These separations in a frequency domain are effective for echo imaging and displacement measurements. 
A
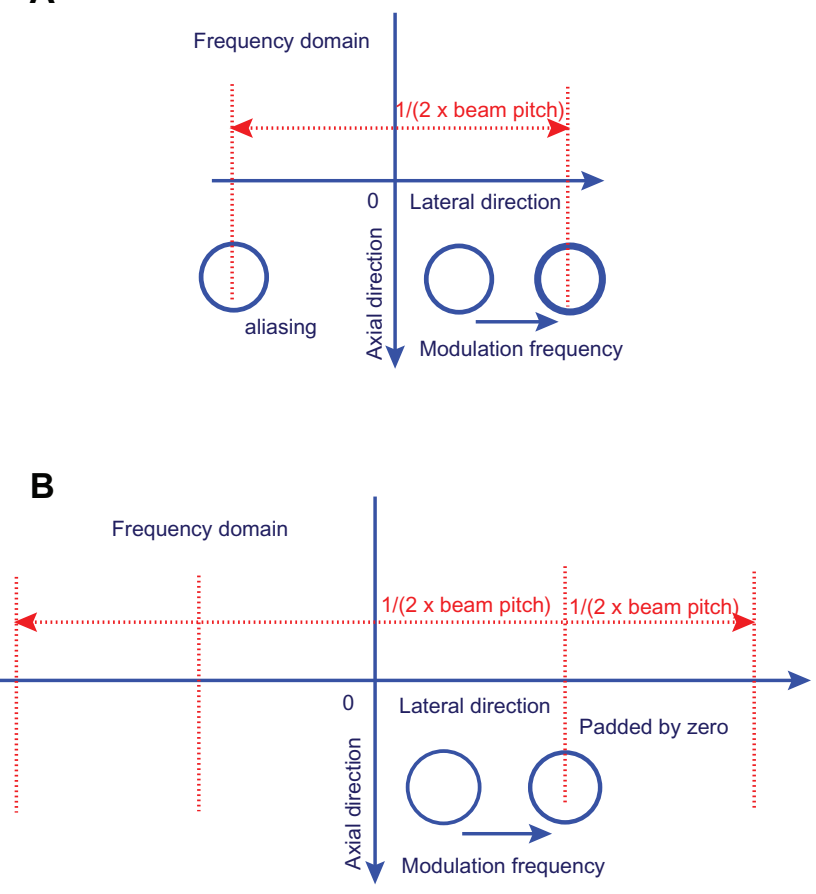

Appendix 2 A) Occurrence of aliasing spectra due to an increase in the lateral frequency by increasing the steering angle. B) Increase in a lateral bandwidth by padding with zeros in a frequency domain.

For LM and ASTA, when a beamforming (transmission/ reception of US) is preformed, after receiving echo signals in SA, or after obtaining steered beams, by rotating the coordinate in the spatial (Appendix 4a) or frequency (Appendix 4b) domain, the

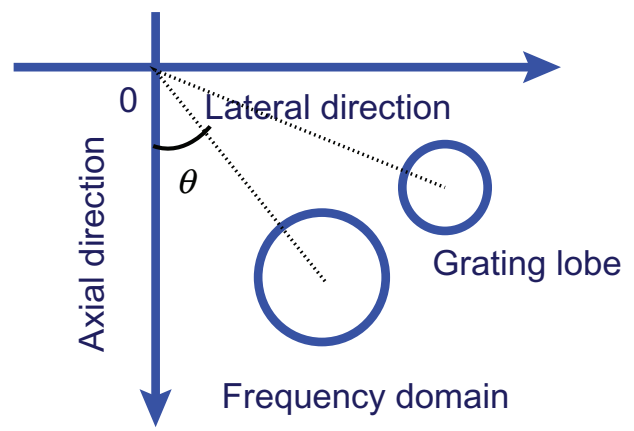

Appendix 3 Separation of overlapped beams in a spatial domain containing grating lobes, side lobes, and signals arriving from other sources.

Reports in Medical Imaging

\section{Publish your work in this journal}

Reports in Medical Imaging is an international, peer-reviewed, open access journal publishing original research, reports, reviews and commentaries on all areas of medical imaging. The manuscript management system is completely online and includes a very quick and fair peer-review system, which is all easy to use. axial, lateral, and elevation frequencies are controlled (relations are shown in Eqs. 2 and 3). ${ }^{46}$ This processing is effective when a sufficiently high lateral frequency cannot be achieved by simply steering a beam due to the decrease in the echo SNR or when a coordinate system must be properly reformed. That is, this rotation is also effective for a non-steering case..$^{42}$ Moreover, for LM, this rotation allows the use of nonsymmetric steering angles with 1D displacement measurement methods. ${ }^{42}$ When performing the coordinate rotation after performing the beamforming, some interpolation method must be applied to the corresponding original rf signals.

\section{A}

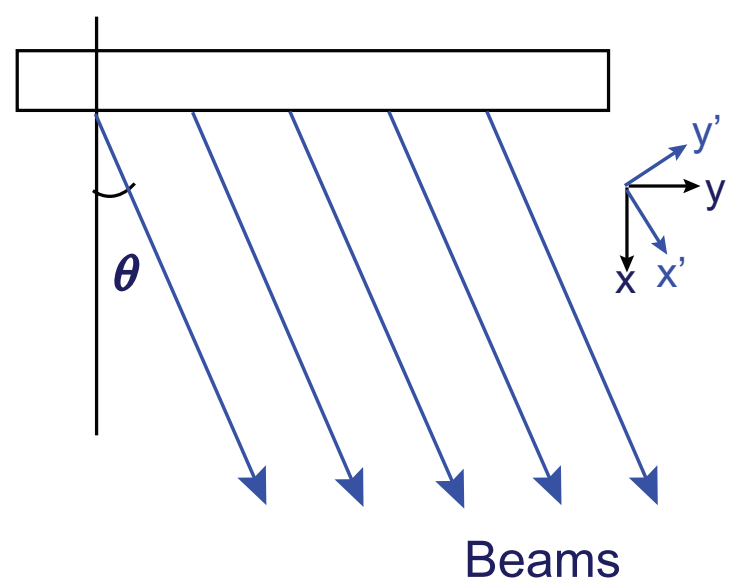

B

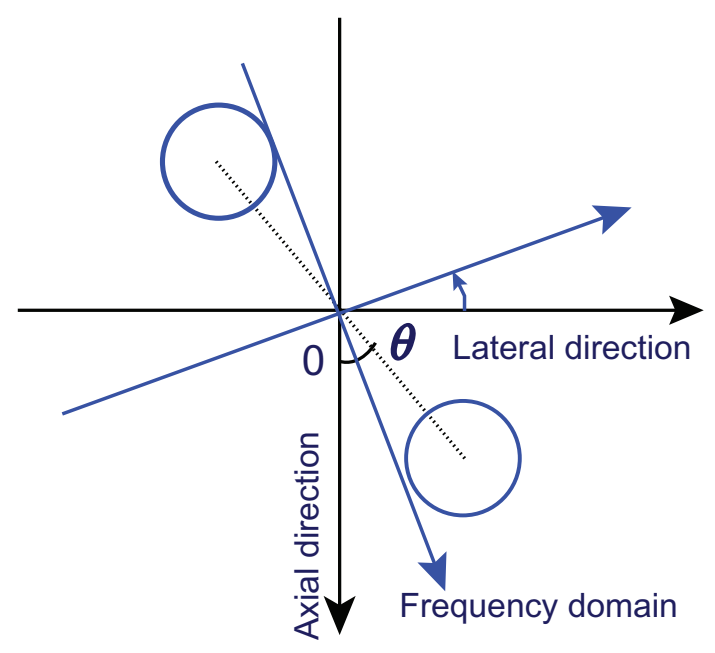

Appendix 4 Rotation of coordinates in spatial (A) and frequency (B) domains.
Visit http://www.dovepress.com/testimonials.php to read real quotes from published authors. 